\title{
Trait-based population dynamics shape the contours of fitness landscapes
}

Andrew Siefert and Daniel C. Laughlin

Department of Botany, University of Wyoming, Laramie, WY 82071

\begin{abstract}
Ecological responses to environmental change are driven by population demography ${ }^{1,2}$, yet traitbased population dynamics are rarely used to project community assemblages ${ }^{3}$. Fitness landscapes describe how selection influences the evolution of species ${ }^{4,5}$ and can be extended to multispecies communities along environmental gradients based on the matching of traits to local environmental conditions ${ }^{6}$. Knowing the contours of fitness landscapes would improve our understanding of how selection shapes ecological communities and would permit predictions of community dynamics in changing environments. However, the shape of fitness landscapes for multispecies communities is unknown because we have lacked a framework for estimating fitness across individuals, phenotypes, and environments. Here we describe a new framework to estimate multispecies fitness landscapes and illustrate model results for trees in temperate forests across a climatic gradient in the eastern United States. Demographic models showed that multivariate phenotypes interact with climate to influence rates of tree growth, survival, and reproduction and generate demographic trade-offs that constrain life history strategies. By integrating trait effects on demographic performance across the life cycle, we estimated the net effect of traits on fitness and reveal the shapes of fitness landscapes driven by temperature. With increasing availability of trait ${ }^{7}$ and demographic data ${ }^{8,9}$, our approach can be applied to any ecological system, providing a pathway to generate mechanistic, general predictions of population and community responses to environmental change.
\end{abstract}

\section{Main}

Fitness landscapes, introduced nearly 100 years ago by Sewall Wright, describe the relationship between multidimensional genotypes ${ }^{10}$ or phenotypes ${ }^{4}$ and fitness. Natural selection is expected to push populations toward peaks in the fitness landscape because individuals with more adaptive traits will survive and reproduce at greater rates ${ }^{11}$. The shape of the fitness landscape not only influences the course of evolution within populations but also the process of community assembly among populations ${ }^{6}$. A multispecies fitness landscape quantifies how phenotypic 
variation among species affects species' fitness, which we define as the population growth rate $(\lambda)^{12}$. These landscapes are not static, but rather change shape because the adaptive value of traits are themselves functions of environmental gradients ${ }^{6}$. Knowing the shape of multispecies fitness landscapes and how they change across environmental gradients would allow us to understand the selective forces that drive community assembly and improve our ability to predict population and community dynamics in changing environments.

Constructing fitness landscapes for entire assemblages of species has been fraught with empirical challenges. The expectation that functional traits influence demographic performance is foundational to trait-based community ecology ${ }^{2}$, but trait-demographic rate relationships are often weak, possibly because these relationships are highly context-dependent ${ }^{13}$. The effect of a single trait on demographic performance depends on 1) other traits of the organism (i.e., trait-bytrait interactions $)^{14}, 2$ ) the life stage or size of the individual (i.e., trait-by-size interactions) ${ }^{15}$, and 3 ) the local environment (i.e., trait-by-environment interactions $)^{6}$. Estimating multispecies fitness landscapes requires empirical measures of fitness and traits on multiple species across environmental gradients. However, many empirical studies that measure fitness are limited to one to few species, and if a study does include multiple species, it is often limited to single demographic rates given the logistical challenges of measuring fitness.

To solve these challenges, we incorporated all these contexts into hierarchical Bayesian models (see extended Methods) to estimate the effects of multidimensional phenotypes and their interaction with size and temperature on survival, growth, and recruitment rates of trees in the eastern US. We combined species' functional trait data from a global database ${ }^{7}$ and demographic data from a national forest inventory (US Forest Service Forest Inventory and Analysis) to estimate multispecies fitness landscapes for trees across a temperature gradient in the eastern United States. We analyzed three traits representing key axes of plant functional strategies ${ }^{16}$. Wood density reflects trade-offs between stem hydraulic efficiency, hydraulic safety, and mechanical strength ${ }^{17}$. SLA reflects the trade-off between the cost of leaf construction and rate of return on investment in carbon and nutrients ${ }^{18}$. Maximum height $(\mathrm{m})$ reflects a trade-off where taller species are better competitors for light but have higher stem construction and maintenance costs and deferred reproduction ${ }^{19}$. We modeled trait effects on growth, survival, and recruitment as three-dimensional Gaussian surfaces that could vary with tree size and local mean annual temperature. Integral projection models ${ }^{20}$ were used to integrate demographic performance 
across the life cycle to quantify the net effects of multidimensional phenotypes on fitness across the temperature gradient.

\section{Trait-demographic rate relationships}

Functional traits influenced tree survival, growth, and recruitment rates, and these effects varied with tree size and mean annual temperature. Tree species with the tallest maximum heights and densest wood survived at the greatest rates across the temperature gradient (Fig. 1a-d; Extended Data Fig. 2a,c), consistent with previous findings in tropical forests ${ }^{21,22}$. Wood density and SLA had an interactive effect on survival, especially of small-diameter trees, such that survival peaked for species with either high wood density and high SLA or low wood density and low SLA (Fig. 1a; Extended Data Fig. 4a).

Species with the tallest maximum heights and lowest wood density grew the fastest, especially in cold sites (Fig. 1g,h; Extended Data Fig. 2d,f) ${ }^{22,23}$. There was a complex interaction between wood density, SLA, and temperature, such that in cold sites growth peaked at low values of wood density and medium to high values of SLA (Fig. 1e), whereas in warm sites there was a ridge of high growth rates in the landscape running from an acquisitive strategy of low wood density and high SLA to a conservative strategy of high wood density and low SLA (Fig 1f; Extended Data Fig. 5a). The positive effect of SLA on growth was strongest in smalldiameter trees (Extended Data Fig. 2e), consistent with theoretical expectations that SLA is most important for performance at the sapling stage, when leaves make up a relatively large proportion of tree biomass ${ }^{15}$, whereas the effect of maximum height was strongest for largediameter trees (Extended Data Fig. 2f).

Species with low wood density exhibited the highest per-capita recruitment rates (i.e., the rate of new recruits reaching the $2.54 \mathrm{~cm}$ diameter threshold per adult tree), particularly in warm sites (Fig 1j,1; Extended Data Fig 3a). There was strong positive correlational selection between wood density and SLA, such that recruitment peaked at a combination of low wood density and low SLA and a combination of high wood density and high SLA, especially in warm sites (Fig 1j; Extended Data Fig 6a). The effects of maximum height and wood density on per-capita recruitment depended on tree diameter. For small-diameter trees, per-capita recruitment was strongly negatively related to wood density and maximum height, whereas for large-diameter trees these effects were weaker (Extended Data Fig. 3a,c). These interactions occurred because species with dense wood and tall maximum height had a larger size at onset of reproduction (Fig. 
2d), consistent with findings in tropical forests ${ }^{23}$ and expectations from game theoretical models ${ }^{19}$.
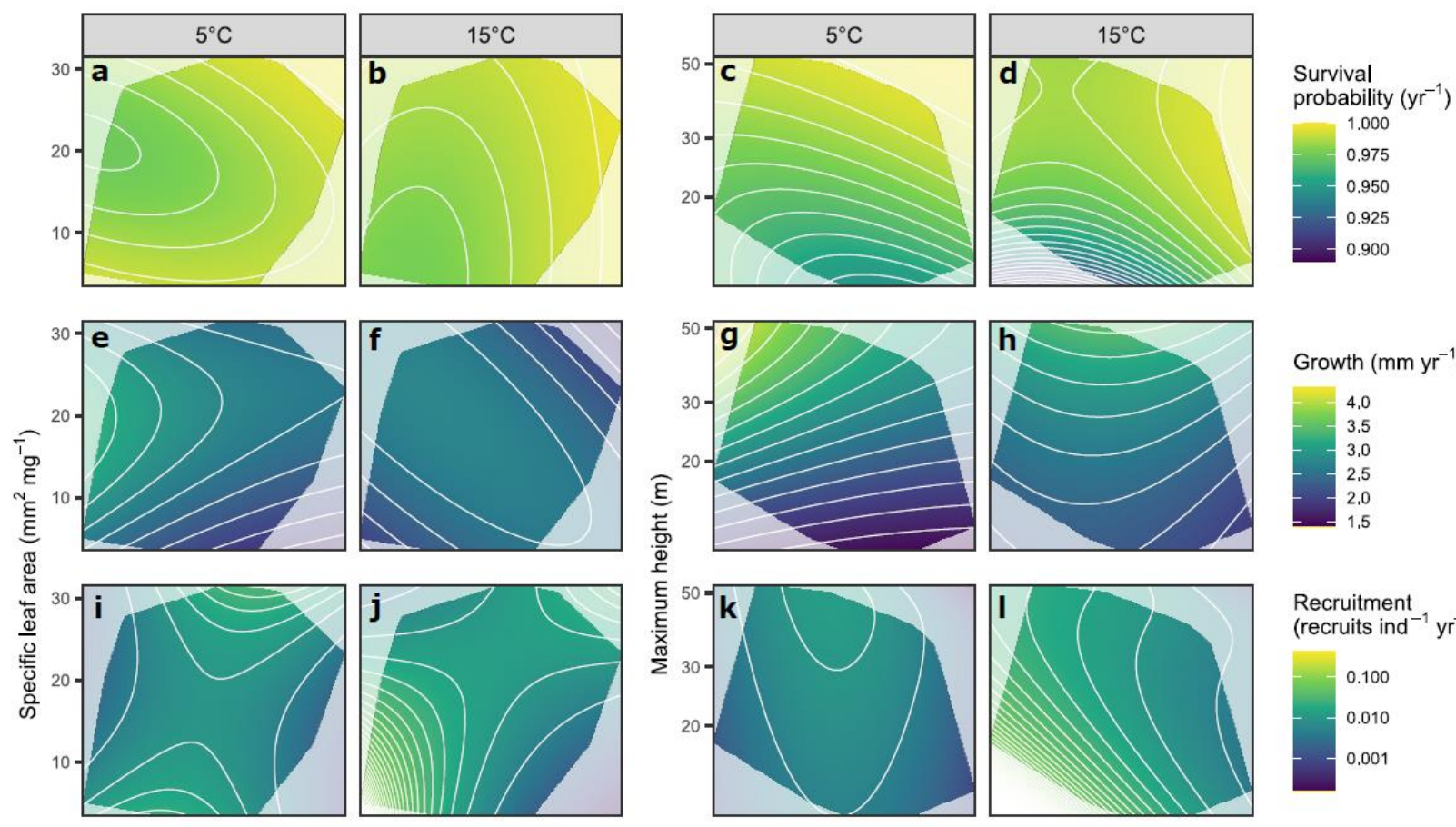

Growth $\left(\mathrm{mm} \mathrm{yr}^{-1}\right)$
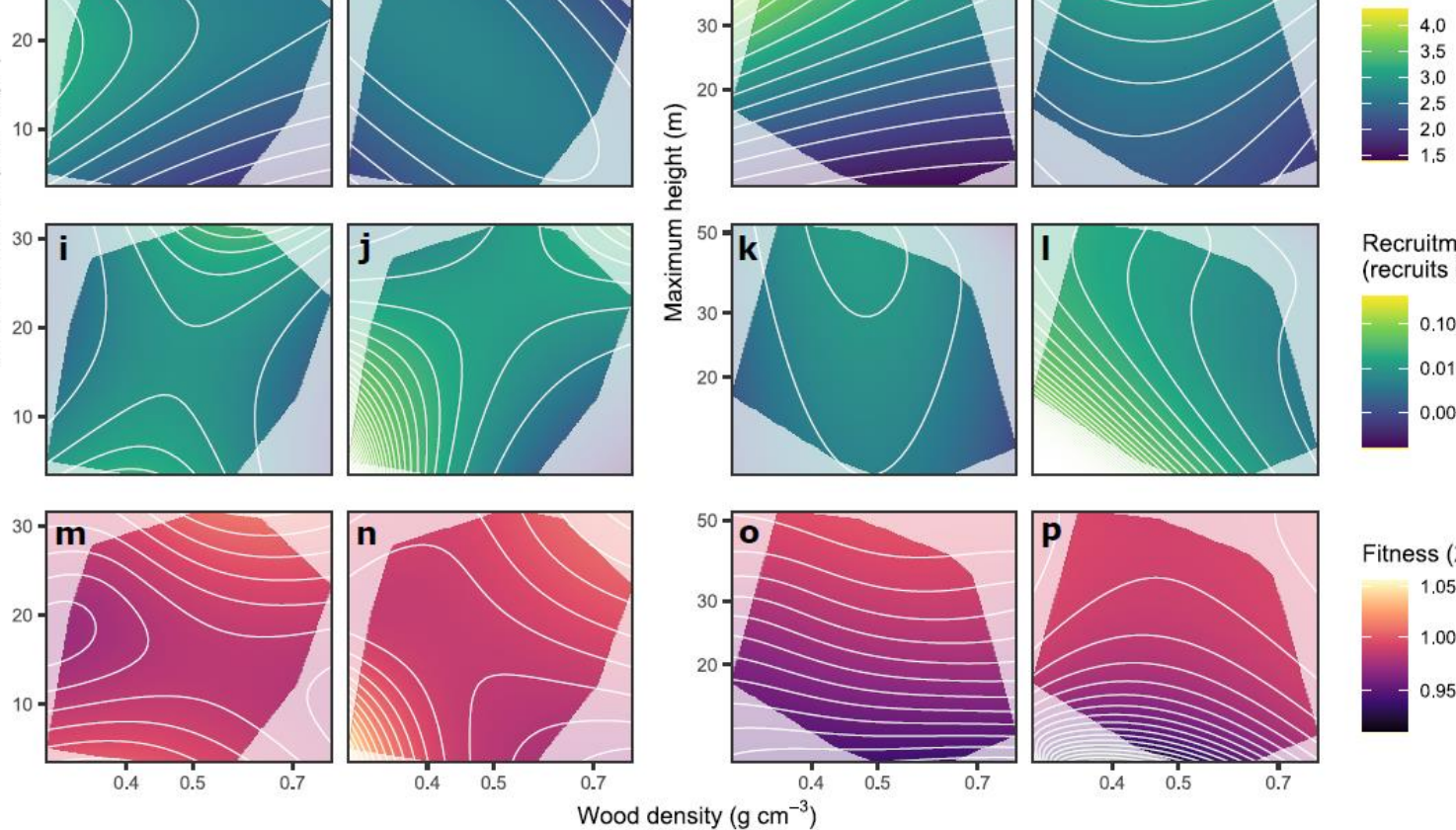

Recruitment (recruits ind ${ }^{-1} \mathrm{yr}^{-1}$ )

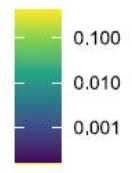

Wood density $\left(\mathrm{g} \mathrm{cm}^{-3}\right)$

Figure 1. Tree performance and fitness landscapes at low and high mean annual temperatures.

Landscapes show expected demographic rates (survival, a-d; diameter growth, e-h; recruitment, i-l) and fitness (population growth rate, $\mathrm{m}-\mathrm{p}$ ) for trees species with different trait combinations. Demographic rate and fitness models included three traits — wood density, specific leaf area (SLA), and maximum heightbut for ease of visualization, landscapes are shown for two traits at a time (wood density and SLA, columns 1-2; wood density and maximum height, columns 3-4) with the third trait held constant at its average value. Demographic rates also vary with tree size, mean annual temperature, and neighbor density in our model. Performance landscapes shown here are for trees with $20 \mathrm{~cm}$ diameter at low $\left(5^{\circ} \mathrm{C}\right.$, columns 1 and 3$)$ or high $\left(15^{\circ} \mathrm{C}\right.$, columns 2 and 4$)$ mean annual temperature and average neighbor density. Fitness landscapes integrate demographic performance across sizes. Grayed areas show regions of trait space not occupied by tree species in our data set. 


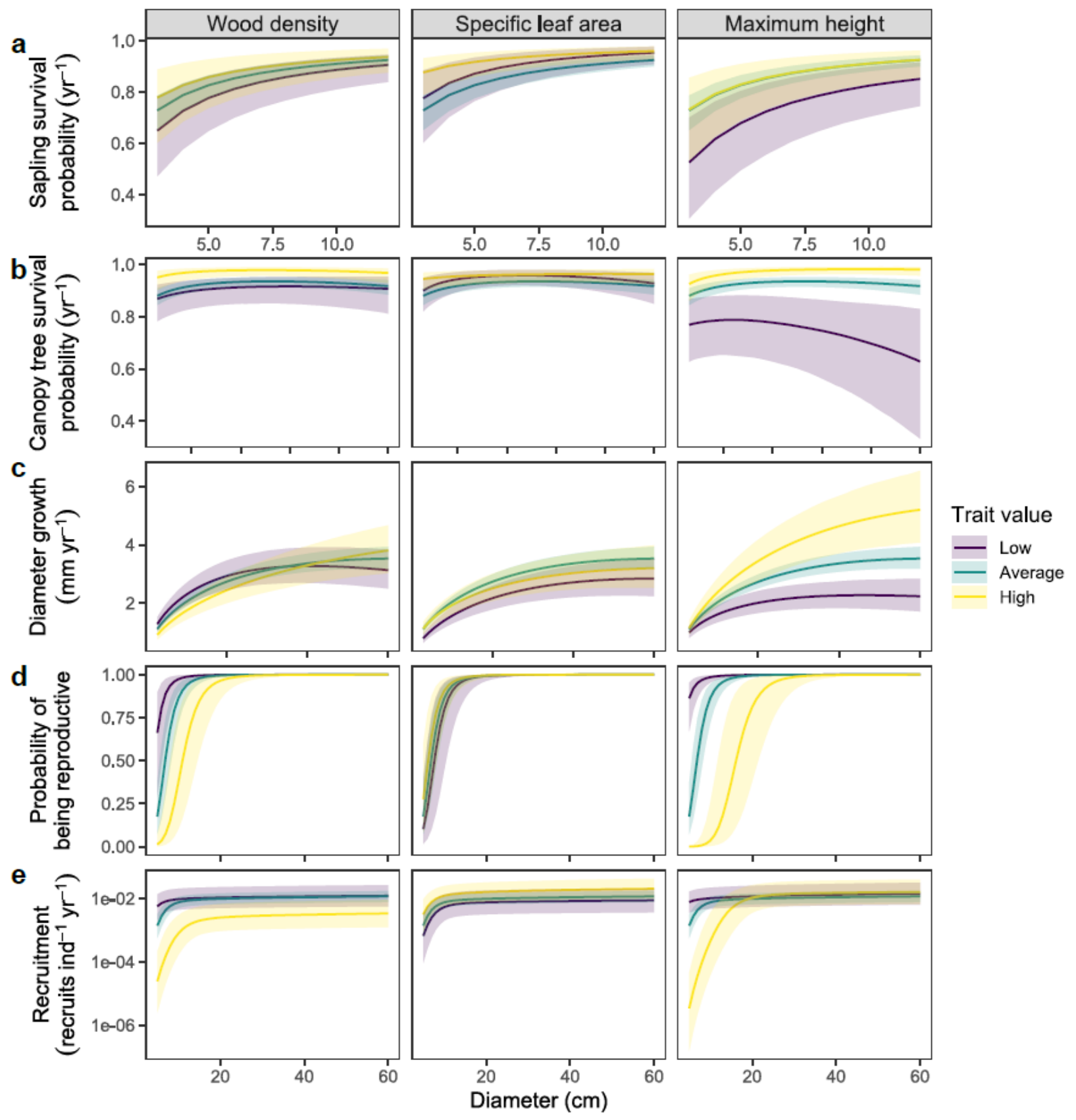

Figure 2. Relationship between tree diameter and demographic performance with respect to

functional traits. Plots show the relationship between tree diameter and expected sapling survival (a), canopy tree survival (b), diameter growth (c), probability of being reproductive (d), and annual per-capita recruitment (e). Trend lines show conditional expectations for trees with different values of wood density (column 1), specific leaf area (column 2), or maximum height (column 3) with other traits, temperature, and neighbor density held constant at their average values. Size-demographic rate relationships are show for average, low (average - 2SD), and high (average + 2SD) values of each trait to illustrate trait-by-size interactions. Shaded areas show $90 \%$ credible intervals. 


\section{Trait-mediated demographic trade-offs}

Life history theory holds that organisms face trade-offs in allocation to different demographic processes (survival, growth, and reproduction) across the life cycle, resulting in demographic trade-offs that constrain life history strategies ${ }^{24}$. Key demographic trade-offs posited for trees include the growth-survival trade-off, which is thought to be strongest at the sapling stage ${ }^{25}$, and the stature-recruitment trade-off, which distinguishes between phenotypes that recruit early in life versus those that exhibit high growth and survival later in life ${ }^{26}$. Functional traits have been shown to be correlated with species' positions along these demographic trade-off axes ${ }^{25,26}$, but the degree to which functional trait variation drives these demographic trade-offs is poorly understood. To address this, we tested whether growth-survival and stature-recruitment tradeoffs emerged from the effects of traits on demographic rates in our models.

We found evidence for a growth-survival trade-off among saplings driven by wood density, where species with low wood density had high sapling growth rates but low survival and species with high wood density had high sapling survival but low growth rates (Fig. 3a). This growth-survival trade-off naturally emerges from the opposing effects of wood density on growth and survival. We also found evidence of a stature-recruitment trade-off mediated by maximum height and wood density. Species with low maximum height and low wood density had high recruitment at small sizes but low growth and survival at larger sizes, whereas species with tall maximum height and dense wood had high growth and survival as large trees but produced few recruits when they were small (Fig. 2b,c,h,i). We did not find evidence of a growth-survival trade-off or stature-recruitment trade-off driven by specific leaf area (Fig 2d-f). 

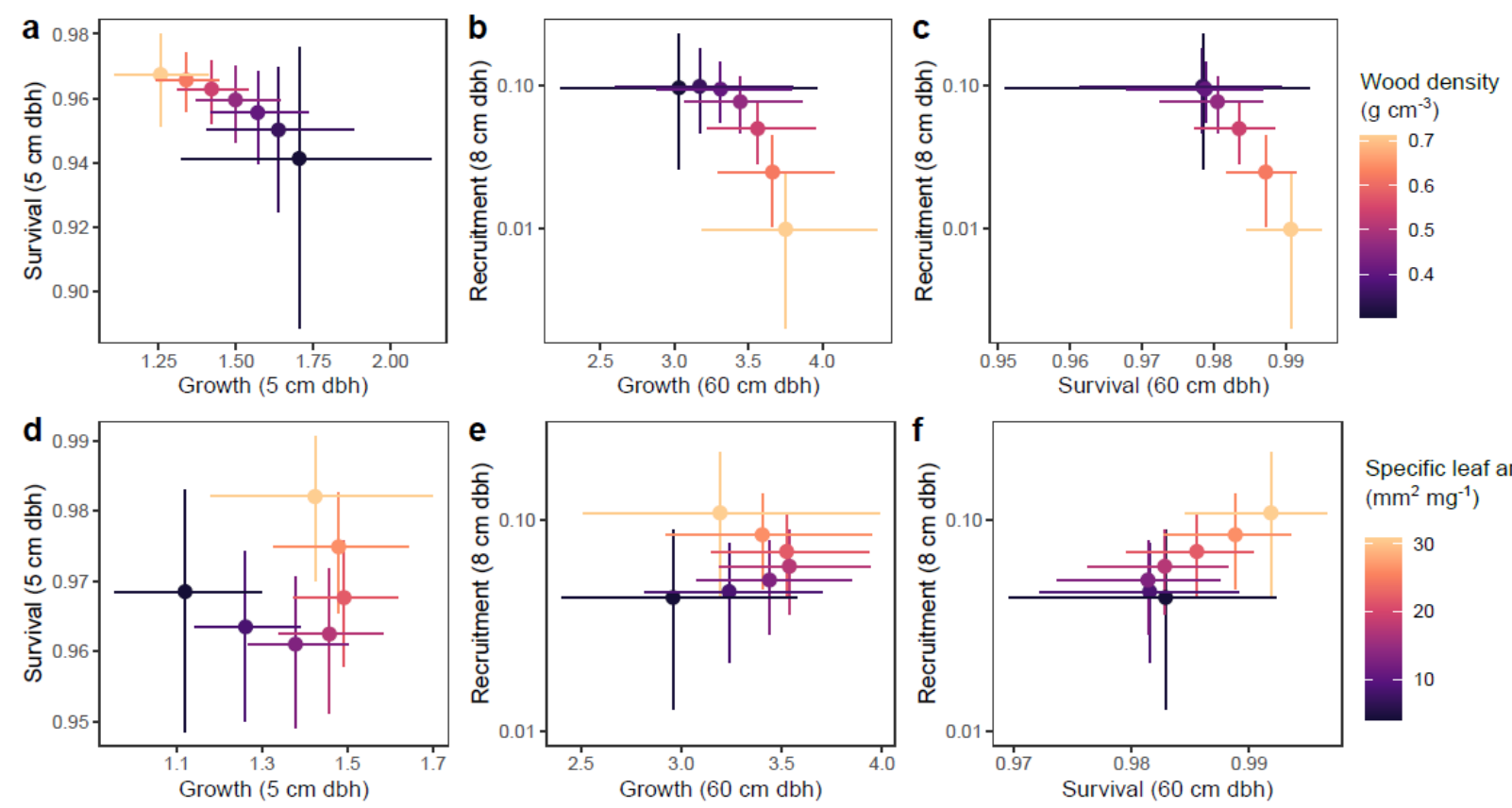

Specific leaf area $\left(\mathrm{mm}^{2} \mathrm{mg}^{-1}\right)$
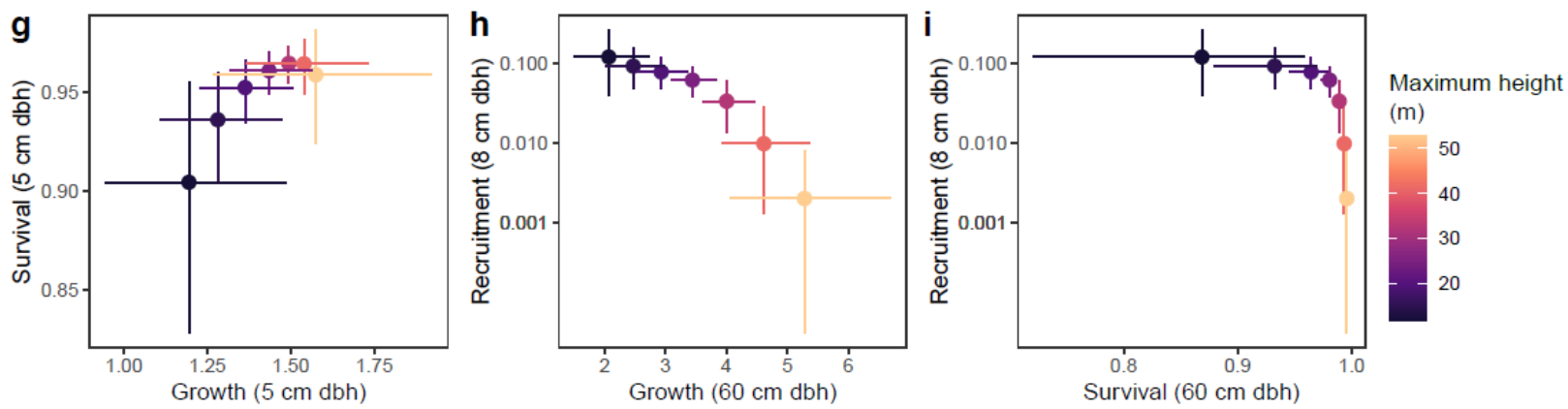

Figure 3. Trait-mediated demographic trade-offs. Points represent predicted demographic rates (error bars show $90 \%$ credible intervals) for trees with varying wood density (a-c), specific leaf area (d-f), or maximum height (g-i), with other model predictors held at their average values. A negative relationship between demographic rates across values of a trait indicates a trait-mediated demographic trade-off. The first column ( $\mathrm{a}, \mathrm{d}, \mathrm{b})$ shows the relationship between predicted growth and survival at $5 \mathrm{~cm}$ diameter, diagnostic of a growth-survival tradeoff in saplings. The second (b,e,h) and third (c,f,i) columns show relationships between growth and survival, respectively, at $60 \mathrm{~cm}$ diameter and recruitment at $8 \mathrm{~cm}$ diameter, diagnostic of a trade-off between recruitment early in life and growth and survival later in life (i.e., stature-recruitment tradeoff). 


\section{Dynamic fitness landscapes}

Population responses to environmental change are driven by demography, yet we have lacked a framework for modeling trait-based population dynamics that integrates all demographic rates. Rather than building individual population models for every species, we built one population model for all species based on traits, individual sizes, temperature, and their interactions. This allowed us to quantify fitness landscapes that describe the net effects of multidimensional phenotypes on fitness across the temperature gradient. Fitness increased with maximum height in all temperatures, with short-statured species having population growth rates $(\lambda)$ well below 1 , especially in colder sites (Fig. 1o-p; Extended Data Fig. 7b,c; Extended Data Fig. 8). The positive overall effect of maximum height on fitness likely results from its positive effects on both growth and survival across the lifespan (Extended Data Fig. 2c,f). Short-statured trees reached reproductive maturity quickly and had high recruitment rates at small diameters (Fig. 2d; Extended Data Fig. 3c), but this had relatively little effect on total fitness because fitness of longlived plants is more sensitive to survival and growth than to reproduction ${ }^{27}$. The low fitness of short-statured species may reflect our choice to only include forests that were at least 20 years old in our data set, as short-statured, early-successional species are likely outcompeted for light by taller, late-successional species by this time ${ }^{28}$. Processes not explicitly included in our models, including gap formation and niche partitioning, may allow short-statured species to persist in mature forests despite their low average fitness ${ }^{29}$.

The net effects of wood density and specific leaf area on fitness were weaker, nonlinear, interactive, and variable across the temperature gradient. The strongest effects of wood density and SLA were in warm sites, where fitness was highest for species with low wood density and low SLA (Fig. 1n; Extended Data Fig 7a), likely due to these species having high sapling survival coupled with extremely high recruitment rates (Extended Data Fig. 4a, 6a). This portion of the trait space is occupied by conifers (e.g., Pinus spp.; Extended Data Fig. 1a). Our finding of high fitness in low-wood density, low-SLA species in warm sites is consistent with previous projections for this region that pine species will increase in abundance in response to climate change this century ${ }^{30}$. Fitness landscapes for wood density and SLA were bimodal, with fitness peaks at both low wood density coupled with low SLA and high wood density coupled with high SLA (Fig. 1m-n; Extended Data Fig. 7a). This appears to be driven by a similar bimodal shape of the performance landscape for sapling survival (Extended Data Fig. 4a). These bimodal and 
relatively flat fitness landscapes provide evidence of alternative functional strategies that yield similar fitness, potentially contributing to the maintenance of functional diversity ${ }^{31}$.

\section{Discussion}

Functional traits influenced demographic performance and fitness of trees across the eastern United States. Tree species with tall maximum height had relatively high survival and grew quickly throughout their life cycle but produced few recruits as young trees, reflecting a strategy of "long-lived pioneers" previously described in tropical trees ${ }^{26}$. The positive effects of maximum height on survival and growth outweighed its negative effects on recruitment, resulting in a generally positive net effect of maximum height on fitness. Species with dense wood had high survival across the life cycle but grew slowly as saplings and produced few recruits, reflecting a slow life history strategy. As a result of these demographic trade-offs, the net effect of wood density on fitness was relatively weak. Wood density also interacted with specific leaf area, producing bimodal fitness landscapes indicating alternative strategies with relatively high fitness ${ }^{31}$. Importantly, whereas trait distribution patterns reflect the aggregated effects of multiple processes, including selection, dispersal, and ecological drift, acting across multiple generations ${ }^{3}$, our estimated fitness landscapes capture the effects of selection in contemporary environments. Quantifying the shape of fitness landscapes advances our understanding of the adaptive value of functional traits across environments and will improve predictions of population and community dynamics in the face of rapid environmental change. Our model estimated changes in fitness landscapes across a temperature gradient, but for the sake of model tractability we did not incorporate frequency-dependent effects of the biotic neighborhood. Our model also did not incorporate the effects of canopy position or spatial and temporal variability in light, which are known to be important drivers of forest dynamics ${ }^{32}$. Future work will extend the models to include temporal changes in light availability and will integrate how fitness landscapes change as a function of the traits of neighbors.

Here we have shown for the first time how to quantify the shape of fitness landscapes for multispecies communities to advance our understanding of the adaptive value of functional traits and to improve predictions of population and community dynamics in the face of rapid environmental change. Selection, the differential fitness of individuals due to differences in phenotype, is the key force driving evolution and community assembly ${ }^{33,34}$. Evolutionary 
biologists have estimated fitness landscapes that capture selection within species ${ }^{5}$, but the shape of fitness landscapes for multispecies communities has remained hidden due to the empirical challenges of estimating fitness across phenotypes of multiple species. Our novel approach overcomes these challenges by integrating trait-based demographic models into a single population projection model to estimate the net effect of multidimensional phenotypes on fitness across species and environments. With this approach it is now possible to empirically estimate fitness landscapes for multispecies communities across environmental gradients, leading to improved understanding of the selective forces that drive community assembly and permitting mechanistic predictions of community dynamics in changing environments. Our approach is flexible and can be applied in any system given the availability of trait and demographic data, which are becoming more widely available due to the proliferation of global databases ${ }^{7-9}$, providing a promising pathway to achieve the long-held goal of making community ecology more general, mechanistic, and predictive.

\section{References}

1. Harper, J. L. A Darwinian approach to plant ecology. J. Ecol. 55, 247-270 (1967).

2. Violle, C. et al. Let the concept of trait be functional! Oikos 116, 882-892 (2007).

3. Laughlin, D. C., Gremer, J. R., Adler, P. B., Mitchell, R. M. \& Moore, M. M. The net effect of functional traits on fitness. Trends Ecol. Evol. 35, 1037-1047 (2020).

4. Simpson, G. G. Tempo and Mode in Evolution. (Columbia University Press, 1944).

5. De Visser, J. A. G. M. \& Krug, J. Empirical fitness landscapes and the predictability of evolution. Nat. Rev. Genet. 15, 480-490 (2014).

6. Laughlin, D. C. \& Messier, J. Fitness of multidimensional phenotypes in dynamic adaptive landscapes. Trends Ecol. Evol. 30, 487-496 (2015).

7. Kattge, J. et al. TRY - a global database of plant traits. Glob. Chang. Biol. 17, 2905-2935 (2011).

8. Salguero-Gómez, R. et al. The compadre Plant Matrix Database: An open online repository for plant demography. J. Ecol. 103, 202-218 (2015).

9. Salguero-Gómez, R. et al. COMADRE: A global data base of animal demography. $J$. Anim. Ecol. 85, 371-384 (2016).

10. Wright, S. The roles of mutation, inbreeding, crossbreeding, and selection in evolution. 
Proc. Sixth Int. Congr. Genet. 1, 356-366 (1932).

11. Lande, R. Natural selection and random genetic drift in phenotypic evolution. Evolution 30, 314-334 (1976).

12. McGraw, J. B. \& Caswell, H. Estimation of individual fitness from life-history data. Am. Nat. 147, 47-64 (1996).

13. Yang, J., Cao, M. \& Swenson, N. G. Why functional traits do not predict tree demographic rates. Trends Ecol. Evol. 33, 326-336 (2018).

14. Lande, R. \& Arnold, S. J. The measurement of selection on correlated characters. Evolution 37, 1210-1226 (1983).

15. Falster, D. S., Duursma, R. A. \& FitzJohn, R. G. How functional traits influence plant growth and shade tolerance across the life cycle. Proc. Natl. Acad. Sci. U. S. A. 115, E6789-E6798 (2018).

16. Díaz, S. et al. The global spectrum of plant form and function. Nature 529, 167-171 (2015).

17. Chave, J. et al. Towards a worldwide wood economics spectrum. Ecol. Lett. 12, 351-66 (2009).

18. Wright, I. J. et al. The worldwide leaf economics spectrum. Nature 428, 821-827 (2004).

19. Falster, D. \& Westoby, M. Plant height and evolutionary games. Trends Ecol. Evol. 18, 337-343 (2003).

20. Ellner, S. P., Childs, D. Z., Rees, M. \& others. Data-Driven Modelling of Structured Populations: A Practical Guide to the Integral Projection Model. (Springer, 2016).

21. Chao, K. J. et al. Growth and wood density predict tree mortality in Amazon forests. $J$. Ecol. 96, 281-292 (2008).

22. Poorter, L. et al. Are functional traits good predictors of demographic rates? Evidence from five neotropical forests. Ecology 89, 1908-1920 (2008).

23. Visser, M. D. et al. Functional traits as predictors of vital rates across the life cycle of tropical trees. Funct. Ecol. 30, 168-180 (2016).

24. Stearns, S. C. Trade-offs in life-history evolution. Funct. Ecol. 3, 259-268 (1989).

25. Wright, S. J. et al. Functional traits and the growth-mortality trade-off in tropical trees. Ecology 91, 3664-3674 (2010).

26. Rüger, N. et al. Beyond the fast-slow continuum: demographic dimensions structuring a 
tropical tree community. Ecol. Lett. 21, 1075-1084 (2018).

27. Franco, M. \& Silvertown, J. A comparative demography of plants based upon elasticities of vital rates. Ecology 85, 531-538 (2004).

28. Falster, D. S. \& Westoby, M. Tradeoffs between height growth rate, stem persistence and maximum height among plant species in a post-fire succession. Oikos 111, 57-66 (2005).

29. Falster, D. S., Brännström, Å., Westoby, M. \& Dieckmann, U. Multitrait successional forest dynamics enable diverse competitive coexistence. Proc. Natl. Acad. Sci. U. S. A. 114, E2719-E2728 (2017).

30. Iverson, L. R. \& Prasad, A. M. Predicting abundance of 80 tree species following climate change in the eastern United States. Ecol. Monogr. 68, 465-485 (1998).

31. Marks, C. O. \& Lechowicz, M. J. Alternative designs and the evolution of functional diversity. Am. Nat. 167, 55-66 (2006).

32. Shugart, H. H. A Theory of Forest Dynamics. The Ecological Implications of Forest Succession Models. (Springer-Verlag, 1984).

33. Darwin, C. The Origin of Species by Means of Natural Selection. (Murray, 1859).

34. Vellend, M. Conceptual synthesis in community ecology. Q. Rev. Biol. 85, 183-206 (2010).

\section{Methods}

\section{Data}

We fit demographic models using data from the US Forest Service Forest Inventory and Analysis (FIA; https://apps.fs.usda.gov/fia/datamart/datamart.html). We included plots on forest land with stand age of at least 20 years in the Northeastern Mixed Forest, Eastern Broadleaf Forest, and Southeastern Mixed Forest ecoregions and "rolling uplands" physiographic class. We excluded plots with evidence of human disturbance. FIA plots are repeatedly censused on cycles whose timing varies by state. For each state, we used the census cycle that included the year 2014, ensuring that data for all states came from a completed census cycle conducted using nationally standardized protocols (established in 1999). We excluded plots with a census interval shorter than 4 years. This left 12,752 plots in 20 states. Each FIA plot consists of four 7.32-m radius subplots in which all trees $\geq 12.7 \mathrm{~cm}$ diameter at breast height (dbh; "canopy trees" hereafter) are censused. Each subplot contains a 2.07-m radius microplot in which saplings of diameter $\geq 2.54$ 
$\mathrm{cm}$ and $<12.7 \mathrm{~cm}$ are censused. We excluded trees that were killed or damaged by human activities during the census interval. Canopy trees and saplings are tagged and tracked between censuses, allowing individual survival and growth to be measured over the census interval. We extracted mean annual temperature data for the years spanning the census interval for each plot from Gridmet ${ }^{35}$.

We extracted trait values for tree species in FIA plots from the TRY plant trait database. We included three traits that are widely measured and linked to important aspects of plant functional and life history strategy ${ }^{16}$ : wood density, specific leaf area (SLA), and maximum height. We joined species trait data with FIA data to create datasets for demographic modeling. Wood density and maximum height were log transformed prior to analysis.

Survival and growth were assessed at the individual level. The relationship between individual size and survival was nonlinear and not well described across the entire range of tree sizes by several functional forms we tried, so we created separate sapling $(<12.7 \mathrm{~cm} \mathrm{dbh})$ and canopy tree $(\geq 12.7 \mathrm{~cm} \mathrm{dbh})$ data sets for survival modeling. We modeled growth of saplings and canopy trees together, using average annual diameter growth rate (change in diameter between the first and second census, divided by the census interval) as the response variable. Recruitment was measured at the plot level as the number of new trees crossing the $2.54-\mathrm{cm}$ threshold during the census interval and being tagged as saplings in the second census (i.e., ingrowth). For each data set (sapling survival, canopy tree survival, growth, and recruitment), we randomly split the data into a training set ( $80 \%$ of plots) for model fitting and test set (20\% of plots) for model evaluation. We excluded plots containing fewer than 10 individuals ( 5 individuals for sapling survival) and species occurring in fewer than 10 plots ( 5 plots for sapling survival). The final training data sets contained: 224,153 trees, 8,837 plots, 94 species (canopy tree survival); 45,249 trees, 4,518 plots, 78 species (sapling survival); 250,768 trees, 9,152 plots, 95 species (growth); 32,891 plot-species observations, 4,099 plots, 83 species (recruitment).

To improve estimates of the relationship between tree size and recruitment, we obtained data on individual size and reproductive status (presence of reproductive structures) from the MASTIF network ${ }^{36}$. We selected data from sites in eastern North America that had at least one species in common with our demography modeling data set. Data were collected between 2002 and 2020, with some trees being measured in multiple years. We excluded observations for 
which reproductive status was unknown, resulting in a data set of 48,082 observations of 27,641 unique trees from 60 species in 34 sites.

\section{Demographic models}

We modeled survival, growth, and recruitment using hierarchical Bayesian models that included terms representing the effects of size, crowding, climate, and traits, as well as species and plot random effects. To capture trait-by-size interactions, the size effects were allowed to vary depending on a species' trait values. To estimate effects of multidimensional phenotypes on performance (i.e., performance landscapes) across the temperature gradient, we modeled trait effects as multivariate Gaussian surfaces whose shape varied with temperature. All predictor variables in the models were scaled to zero mean and unit variance unless otherwise noted.

\section{Survival}

The relationship between size and survival was not well described across the entire range of tree sizes by several functional forms we tried, so we built separate models for saplings $(\mathrm{dbh}<12.7$ $\mathrm{cm})$ and canopy trees $(\mathrm{dbh} \geq 12.7 \mathrm{~cm})$. For both saplings and canopy trees, we modeled survival as:

$\operatorname{survival}_{i} \sim \operatorname{Bernoulli}\left(p_{i}\right)$

$p_{i}=\left(\frac{S_{i}}{1+S_{i}}\right)^{R I_{i}}$

where $p_{i}$ is the expected survival probability of individual $i$ and $R I_{i}$ is the census interval for individual $i$, in years. $S_{i}$ determines the expected 1-year survival probability of individual $i$. Building on models of Canham et al. ${ }^{37,38}$, we modeled survival as a multiplicative function of terms representing the potential survival rate of species $s$ in plot $p$ when all other predictors are at their average values $\left(P S_{s p}\right)$ and effects of size, crowding, climate, and traits:

$S_{i s p}=P S_{s p} \times$ size $_{i s} \times$ crowding $_{i s} \times$ climate $_{s p} \times$ trait $_{s p}$

Potential survival was modeled as:

$P S_{s p}=\exp \left(\alpha_{\mathrm{PS}}+\gamma_{\mathrm{PS}_{s}}+\gamma_{\mathrm{PS}_{p}}\right)$ 
where $\alpha_{\mathrm{PS}}$ determine the average potential survival, $\gamma_{\mathrm{PS}_{s}}$ is a random effect for species $s$, and $\gamma_{\mathrm{PS}_{p}}$ is a random effect for plot $p$.

For canopy tree survival, we modeled the ontogenetic effect of tree size as:

size $_{i s}=\exp \left(\beta_{\text {size } 1_{s}} \log \left(\mathrm{dbh}_{i}\right)+\beta_{\text {size } 2_{s}} \mathrm{dbh}_{i}\right)$

where size $e_{i s}$ is the ontogenetic effect of size on the survival of individual $i$ of $\operatorname{species} s, \mathrm{dbh}_{i}$ is the diameter of individual $i$ at the beginning of the census interval, and $\beta_{\operatorname{size} 1_{s}}$ and $\beta_{\text {size } 2_{s}}$ are coefficients defining the shape of the size curve for species $s$. The function is hump-shaped (survival probability increases with size up to an optimum size, beyond which survival decreases) when $\beta_{\text {size } 1}$ is positive and $\beta_{\text {size2 }}$ is negative.

For saplings, we assumed that survival increased monotonically with size:

$\operatorname{size}_{i s}=\mathrm{dbh}_{i}{ }^{\beta \text { size1s }_{s}}$

The parameters of the size functions were modeled as functions of traits, allowing for trait-bysize interactions, with species random effects:

$\beta_{\text {size } 1_{s}}=\beta_{\text {size } 1}+\boldsymbol{\delta}_{\text {size } 1}$ trait $_{s}+\gamma_{\text {size } 1_{s}}$

$\beta_{\text {size2 }_{s}}=\beta_{\text {size } 2}+\boldsymbol{\delta}_{\text {size } 2}$ trait $_{s}+\gamma_{\text {size } 2 s_{s}}$

where $\beta_{\text {size1 }}$ and $\beta_{\text {size1 }}$ are average size effects, $\boldsymbol{\delta}_{\text {size1 }}$ and $\boldsymbol{\delta}_{\text {size2 } 2}$ are vectors of trait-by-size interaction coefficients, trait ${ }_{s}$ is the vector of trait values for species $s$, and $\gamma_{\operatorname{size} 1_{s}}$ and $\gamma_{\operatorname{size} 2_{s}}$ are species random effects.

The effect of crowding was modeled as a power law function of neighbor basal area:

crowding $_{i s}=\mathrm{BA}_{i}{ }_{\text {crowd }_{s}}$

where crowding $_{i s}$ is the effect of crowding on the survival of individual $i$ of species $s, \mathrm{BA}_{i}$ is the total basal area of canopy trees in the same plot as individual $i$, and $\beta_{\text {crowd }_{S}}$ defines the response to crowding of species $s$, modeled as:

$\beta_{\text {crowd }_{s}}=\beta_{\text {crowd }}+\gamma_{\text {crowd }_{s}}$ 
where $\beta_{\text {crowd }}$ is the average crowding response and $\gamma_{\text {crowd }_{s}}$ is the random effect for species $s$.

The effect of mean annual temperature was modeled as:

climate $_{s p}=\exp \left(\beta_{\operatorname{clim}_{s}} \mathrm{MAT}_{p}\right)$

where $\mathrm{MAT}_{p}$ is the mean annual temperature at plot $p$ and $\beta_{\operatorname{clim}_{s}}$ defines the growth response to temperature of species $s$, modeled as:

$\beta_{\operatorname{clim}_{s}}=\beta_{\text {clim }}+\gamma_{\operatorname{clim}_{s}}$

where $\beta_{\text {clim }}$ is the average temperature response and $\gamma_{\operatorname{clim}_{s}}$ is the random effect for species $s$. The effect of traits was modeled as a Gaussian (exponentiated quadratic) surface ${ }^{39}$ :

trait $_{s p}=\exp \left(\boldsymbol{\beta}_{\operatorname{dir}_{p}}^{\top}\right.$ trait $_{s}+$ trait $_{s}^{\top} \beta_{\text {nonlin }_{p}}$ trait $\left._{s}\right)$

where trait $_{s p}$ is the effect of traits on the survival probability of species $s$ in plot $p, \boldsymbol{\beta}_{\operatorname{dir}_{p}}$ is a vector of directional (linear) performance gradients in plot $p$, and $\boldsymbol{\beta}_{\text {nonlin }_{p}}$ is a matrix of nonlinear performance gradients in plot $p^{40}$. The diagonal elements of $\boldsymbol{\beta}_{\text {nonlin }}$ measure the strength of stabilizing (if $\beta$ is negative) or disruptive (if $\beta$ is positive) selection for each trait, and the off-diagonal elements measure the strength of correlational selection between trait pairs ${ }^{40}$. Positive correlational selection means that performance is maximized by having either high or low values of both traits. Negative correlational selection means that performance is maximized by having a high value of one trait and low value of the other trait. This function can produce performance landscapes of various shapes, including (when viewed in 2 dimensions) a peak, a saddle, a ridge, or a slope.

To allow performances surfaces to vary across the temperature gradient, the performance surface parameters (elements of $\boldsymbol{\beta}_{\text {dir }}$ and $\boldsymbol{\beta}_{\text {nonlin }}$ ) were modeled as linear functions of mean annual temperature:

$$
\begin{aligned}
& \beta_{\operatorname{dir}_{t, p}}=\beta_{\operatorname{dir}_{t}}+\delta_{\text {dir }_{t}} \mathrm{MAT}_{p} \\
& \beta_{\text {nonlin }_{t u, p}}=\beta_{\text {nonlin }_{t u}}+\delta_{\text {nonlin }_{t u}} \mathrm{MAT}_{p}
\end{aligned}
$$


where $\beta_{\mathrm{dir}_{t, p}}$ is the directional selection coefficient for trait $t$ in plot $p, \beta_{\text {nonlin }_{\text {tup }}}$ is the stabilizing/disruptive selection coefficient for trait $t$ (if $t=u$ ) or correlational selection coefficient for traits $t$ and $u$ (if $t \neq u$ ) in plot $p$. $\beta_{\operatorname{dir}_{t}}$ and $\beta_{\text {nonlin }_{t u}}$ are the performance gradients in a plot with average temperature, and $\delta_{\operatorname{dir}_{t}}$ and $\delta_{\text {nonlin }_{t u}}$ describe how the trait effects change along the temperature gradient (i.e., trait-by-environment interactions).

\section{Growth}

Because growth rates could be zero or positive (we excluded negative growth values as we assume they resulted from damage), we modeled growth as a hurdle Gamma distribution:

$\operatorname{Pr}\left(\operatorname{growth}_{i}\right)= \begin{cases}\theta_{i} & \text { if } \operatorname{growth}_{i}=0 \\ \left(1-\theta_{i}\right) * \operatorname{Gamma}\left(G_{i} \beta, \beta\right) & \text { if } \operatorname{growth}_{i}>0\end{cases}$

where $\theta_{i}$ is the probability of zero growth for individual $i, G_{i}$ is the expected annual growth rate of individual $i$, and $\beta$ is the rate parameter of the Gamma distribution. We assumed that both parts of the hurdle model (probability of zero growth and probabilities of positive growth values) are determined by the same processes. Therefore, instead of modeling them independently as commonly done in hurdle models, we modeled $\theta$ as a function of $G$ :

$\theta_{i}=\frac{1}{1+e^{-\left(z-G_{i}\right)}}$

where of $z$ is an estimated parameter. In this formulation, individuals with high expected growth $\left(G_{i}\right)$ have a low probability of zero growth.

We modeled expected growth as a multiplicative function of terms representing the potential growth rate when all predictors are at their average values $\left(P G_{s p}\right)$ and effects of size, crowding, climate, and traits:

$G_{i s p}=P G_{s p} \times$ size $_{i s} \times$ crowding $_{i s} \times$ climate $_{s p} \times$ trait $_{s p}$

Potential growth was modeled as:

$P G_{s p}=\exp \left(\alpha_{\mathrm{PG}}+\gamma_{\mathrm{PG}_{s}}+\gamma_{\mathrm{PG}_{p}}\right)$ 
where $\alpha_{\mathrm{PG}}$ is the overall average, $\gamma_{\mathrm{PG}_{s}}$ is a random effect for species $s$, and $\gamma_{\mathrm{PG}_{p}}$ is a random effect for plot $p$.

The terms for effects of size, crowding, climate, and traits on growth rate had the same form as the corresponding terms in the canopy survival model described above.

\section{Recruitment}

We modeled plot-level recruitment as a negative binomial distribution:

recruitment $_{s p}=\operatorname{NegBinomial}\left(\operatorname{recr}_{s p} * R I_{p}, \phi\right)$

where $\operatorname{recr}_{s p}$ is the expected annual recruitment of species $s$ in plot $p, R I_{p}$ is the recensus interval of plot $p$ in years, and $\phi$ is an estimated parameter that controls overdispersion.

The expected annual recruitment rate of species $s$ in plot $p$ was determined by summing the expected per-capita annual recruitment rates of censused trees in the plot:

$\operatorname{recr}_{s p}=\sum_{i=1}^{n} R_{i s p}$

where $R_{k s p}$ is the annual production of new recruits by tree $i$ of species $s$ in plot $p$. Similar to survival and growth, we modeled expected per-capita recruitment as a multiplicative function of terms representing the potential recruitment rate and effects of size, crowding, climate, and traits:

$R_{i s p}=P R_{s p} \times$ size $_{i s} \times$ crowding $_{i s} \times$ climate $_{p s} \times$ trait $_{s p}$

Potential recruitment was modeled as:

$P R_{s p}=\exp \left(\alpha_{\mathrm{PR}}+\gamma_{\mathrm{PR}_{s}}+\gamma_{\mathrm{PR}_{p}}\right)$

where $\alpha_{\mathrm{PR}}$ is the overall average, $\gamma_{\mathrm{PR}_{s}}$ is a random effect for species $s$, and $\gamma_{\mathrm{PR}_{p}}$ is a random effect for plot $p$.

The size effect was modeled as the product of a term representing the effect of size on the probability of being reproductive and a term representing the effect of size on per-capita production of recruits, conditional on being reproductive ${ }^{41,42}$ :

$$
\operatorname{size}_{i s}=\operatorname{logit}^{-1}\left(\beta_{\text {repro }_{s}} *\left(\log \left(\operatorname{dia}_{i}\right)-D_{s}\right)\right) \times \operatorname{dia}_{i}{ }^{\beta_{r e c r}}
$$


where size $_{i s}$ is the ontogenetic effect of size on production of new recruits by individual $i$ of species $s, \operatorname{dia}_{i}$ is the diameter of individual $i$ (in $\mathrm{cm} / 30$ ), $\beta_{\text {repros }_{s}}$ is the slope of the effect of size on the probability of being reproductive for species $s, D_{s}$ is the log-diameter at which individuals of species $s$ have a $50 \%$ chance of being reproductive, and $\beta_{\text {recr }}$ defines the effect of size on per-capita production of new recruits for species $s$.

The parameters of this size function varied among species as a function of species' trait values, allowing trait-by-size interactions:

$D_{s}=D+\boldsymbol{\delta}_{\mathrm{D}}$ trait $_{s}+\gamma_{\mathrm{D}_{s}}$

$\beta_{\text {repro }_{s}}=\exp \left(\beta_{\text {repro }}+\boldsymbol{\delta}_{\text {repro }}\right.$ trait $\left._{s}+\gamma_{\text {repro }_{s}}\right)$

$\beta_{\text {recr }_{s}}=\exp \left(\beta_{\text {recr }}+\boldsymbol{\delta}_{\text {recr }}\right.$ trait $\left._{s}+\gamma_{\text {recr }_{s}}\right)$

where $D, \beta_{\text {repro, and }} \beta_{\text {recr }}$ describe size function parameters for species with average trait values, the $\boldsymbol{\delta}$ 's are vectors of trait effects on the parameters (trait-by-size interactions), and the $\gamma$ 's are species random effects.

The parameters describing the effect of size on the probability of being reproductive were estimated jointly using the plot-level FIA recruitment data and individual-level size and reproductive status data for trees in the MASTIF data set. Reproductive status of MASTIF trees was modeled as:

$\operatorname{repro}_{i} \sim \operatorname{Bernoulli}\left(p r_{i}\right)$

$p r_{j q s p}=\operatorname{logit}{ }^{-1}\left(\beta_{\text {repro }_{s}} *\left(\log \left(\operatorname{dia}_{i}\right)-D_{s}\right)+\gamma_{\text {site }_{p}}+\gamma_{\text {tree }_{q}}\right)$

where $p r_{j q s p}$ is the probability of being reproductive for observation $j$ of tree $q$ (some trees were observed in multiple years) of species $s$ in site $p$, and $\gamma_{\text {site }_{p}}$ and $\gamma_{\text {tree }_{q}}$ are random effects for site and tree, respectively. The site and tree random effects are assumed to be drawn from normal distributions with mean of zero and standard deviation $\tau_{\text {site }}$ and $\tau_{\text {tree, }}$, respectively.

The effect of crowding on per-capita recruitment was modeled as a function of canopy and sapling neighbor basal area:

crowding $_{i s}=\mathrm{BA}_{\mathrm{can}_{i}} \beta_{\mathrm{can}_{s}} \times \mathrm{BA}_{\mathrm{sap}_{i}} \beta_{\mathrm{sap}_{s}}$ 
were $\mathrm{BA}_{\mathrm{can}_{i}}$ and $\mathrm{BA}_{\mathrm{sap}_{i}}$ are the total basal areas of canopy trees and saplings, respectively, in the same plot as individual $i$, and $\beta_{\mathrm{can}_{s}}$ and $\beta_{\mathrm{sap}_{s}}$ define the response of species $s$ to crowding by canopy trees and saplings, respectively. Species' responses to crowding were modeled as:

$\beta_{\mathrm{can}_{s}}=\beta_{\text {can }}+\gamma_{\mathrm{can}_{s}}$
$\beta_{\mathrm{sap}_{s}}=\beta_{\mathrm{sap}}+\gamma_{\mathrm{sap}_{s}}$

where $\beta_{\text {can }}$ and $\beta_{\text {sap }}$ are average responses to crowding by canopy trees and saplings, respectively, and the $\gamma$ 's are species random effects.

The terms for climate and trait effects on recruitment had the same form as the corresponding terms in the survival and growth models.

For each model, plot-level random effects were assumed to be drawn from a normal distribution with mean of zero and standard deviation $\tau_{\text {plot }}$. Species-level random effects were assumed to be drawn from a multivariate normal distribution with means of zero and covariance matrix $\Sigma$ :

$\boldsymbol{\Sigma}=\operatorname{diag}\left(\boldsymbol{\tau}_{\text {species }}\right) \boldsymbol{L} \boldsymbol{L}^{\top} \operatorname{diag}\left(\boldsymbol{\tau}_{\text {species }}\right)$

where $\boldsymbol{\tau}_{\text {species }}$ is a vector of standard deviations of species random effects and $\boldsymbol{L}$ is the Cholesky factor of the correlation matrix of species random effects.

For each model, we assigned weakly informative priors that constrained predicted demographic rates to biologically realistic values, as assessed by simulating data from the prior distributions. Given the amount of data used to fit the models, the priors had negligible effect on the posterior parameter estimates but aided in model convergence. We obtained posterior samples using Hamiltonian Monte Carlo implemented in $\operatorname{Stan}^{43}$. For each model, we ran 4 chains with 2,000 iterations, 1,000 of which were warmup. We assessed model convergence using the $\hat{R}$ statistic $^{44}$ (values for all parameters were $\leq 1.02$ ).

\section{Fitness estimates}

To construct fitness landscapes, we used integral projection models (IPMs) ${ }^{20}$ to estimate asymptotic population growth rates $(\lambda)$ for multidimensional phenotypes across the temperature gradient. The IPMs combine information about individuals' size-specific survival, growth, and 
recruitment rates to project population dynamics ${ }^{45}$. An IPM describes how the size $(z)$ distribution of individuals at time $t, n_{t}(z)$, changes over one time step (year):

$n_{t+1}\left(z^{\prime}\right)=\int_{\Omega} K\left(z^{\prime}, z\right) n_{t}(z)$

where $n_{t+l}\left(z^{\prime}\right)$ is the size distribution at time $t+1, \Omega$ denotes the possible range of individual sizes, and the kernel $K\left(z^{\prime}, z\right)$ describes size transitions through survival, growth, and reproduction:

$K\left(z^{\prime}, z\right)=s(z) G\left(z^{\prime}, z\right)+F\left(z^{\prime}, z\right)$

where $s(z)$ is the survival probability dependent on initial size $z, G\left(z^{\prime}, z\right)$ describes the probability of growing from size $z$ to $z^{\prime}$, conditional on having survived, and $F\left(z^{\prime}, z\right)$ describes the size distribution of new recruits produced by an individual of size $z$.

We constructed kernels for hypothetical populations with different combinations of trait and environmental values using the demographic rate models described above, with crowding effects held constant at their average value and species and plot random effects set to zero. For the survival kernel, $s(z)$, we used the sapling survival model for individuals with size $z<12.7 \mathrm{~cm}$ and the canopy survival model for individuals with size $\geq 12.7 \mathrm{~cm}$. We constructed the recruitment kernel $F\left(z^{\prime}, z\right)$ as the product of the number of new recruits produced annually by an individual of size $z$, estimated using our recruitment model, and the size distribution of new recruits, which we modeled as a Gamma distribution fit to all recruits in the data set. It is possible that traits, environment, or initial size could affect recruit size, but we chose not to model these effects since simulations showed that the recruit size distribution had a negligible effect on projected population growth rates.

We implemented the IPMs using numerical integration (midpoint rule) ${ }^{20}$. This method discretizes the kernel by dividing the size range into a large number of evenly-sized bins and evaluating the kernel at the midpoint of each bin, effectively generating a large matrix population model. We used a size range from 2.5 to $100 \mathrm{~cm}$ and $1000 \mathrm{bins}$, resulting in a $1000 \mathrm{x} 1000$ transition matrix. A matrix this large is necessary due to the large size range and small growth increments of trees. We confirmed that 1000 bins were enough to produce stable numerical results. To avoid "eviction" of individuals with predicted sizes greater than $100 \mathrm{~cm}^{20}$, we treated the largest size bin as a discrete size class that included all individuals with predicted sizes greater than $100 \mathrm{~cm}$. The growth and recruit size distributions are probability distributions and 
should thus integrate to one (or sum to one in the discretized version) for any initial size $z$, but discretizing the kernels resulted in this not being true in all cases. To correct this, for each initial size class $z_{i}$ we divided the probabilities of growing into (or new recruits being within) each size class $z_{j}$ by the sum of those probabilities, thus ensuring that the growth and recruit size probabilities for any initial size $z_{i}$ summed to one.

The population growth rate $(\lambda)$, our estimate of fitness, was extracted as the dominant eigenvalue of the discretized kernel ${ }^{20}$. To estimate fitness landscapes, we constructed kernels and extracted population growth rates for a grid of trait values covering the observed trait space at each of three mean annual temperatures $\left(5,10\right.$, and $\left.15^{\circ} \mathrm{C}\right)$. To quantify expected $\lambda$ values, we constructed kernels using posterior mean parameter values from the demographic rate models. To estimate uncertainty in $\lambda$, we constructed kernels using 800 samples from the posteriors of the demographic rate models and calculated $90 \%$ credible intervals.

\section{Data availability}

The data that support the findings of this study are available on GitHub, http://github.com/andrewsiefert/treescapes.

\section{Code availability}

The code used to generate the results reported in this paper are available on GitHub, http://github.com/andrewsiefert/treescapes.

\section{Methods references}

35. Abatzoglou, J. T. Development of gridded surface meteorological data for ecological applications and modelling. Int. J. Climatol. 131, 121-131 (2013).

36. Clark, J. S., Nuñez, C. L. \& Tomasek, B. Foodwebs based on unreliable foundations: spatiotemporal masting merged with consumer movement, storage, and diet. Ecol. Monogr. 89, e01381 (2019).

37. Canham, C. D. \& Murphy, L. The demography of tree species response to climate: sapling and canopy tree growth. Ecosphere 7, e01474 (2016).

38. Canham, C. D. \& Murphy, L. The demography of tree species response to climate: sapling and canopy tree survival. Ecosphere 8, e01701 (2017). 
39. Lande, $\mathrm{R}$. The genetic covariance between characters maintained by pleiotropic mutations. Genetics 94, 203-215 (1980).

40. Arnold, S. J. Performance surfaces and adaptive landscapes. Integr. Comp. Biol. 43, 367375 (2003).

41. Clark, J. S., LaDeau, S. \& Ibanez, I. Fecundity of trees and the colonization-competition hypothesis. Ecol. Monogr. 74, 415-442 (2004).

42. Ribbens, E., Silander, J. A. \& Pacala, S. W. Seedling recruitment in forests: calibrating models to predict patterns of tree seedling dispersion. Ecology 75, 1794-1806 (1994).

43. Stan Development Team. RStan: the R interface to Stan. R package version 2.21.2.

44. Vehtari, A., Gelman, A., Simpson, D., Carpenter, B. \& Bürkner, P. C. Ranknormalization, folding, and localization: An improved $\hat{R}$ for assessing convergence of MCMC. Bayesian Anal. 16, 667-718 (2021).

45. Merow, C. et al. Advancing population ecology with integral projection models: a practical guide. Methods Ecol. Evol. 5, 99-110 (2014).

\section{Acknowledgements}

We thank Stephen Ellner for modelling feedback and advice. This study was funded by the National Science Foundation EPSCOR Grant \#2019528.

\section{Author contributions}

AS and DCL conceived the study. AS built the model with input from DCL. AS and DCL wrote the manuscript

\section{Competing interest declaration}

The authors declare no competing interests.

\section{Additional information}

Supplementary Information is available for this paper. 

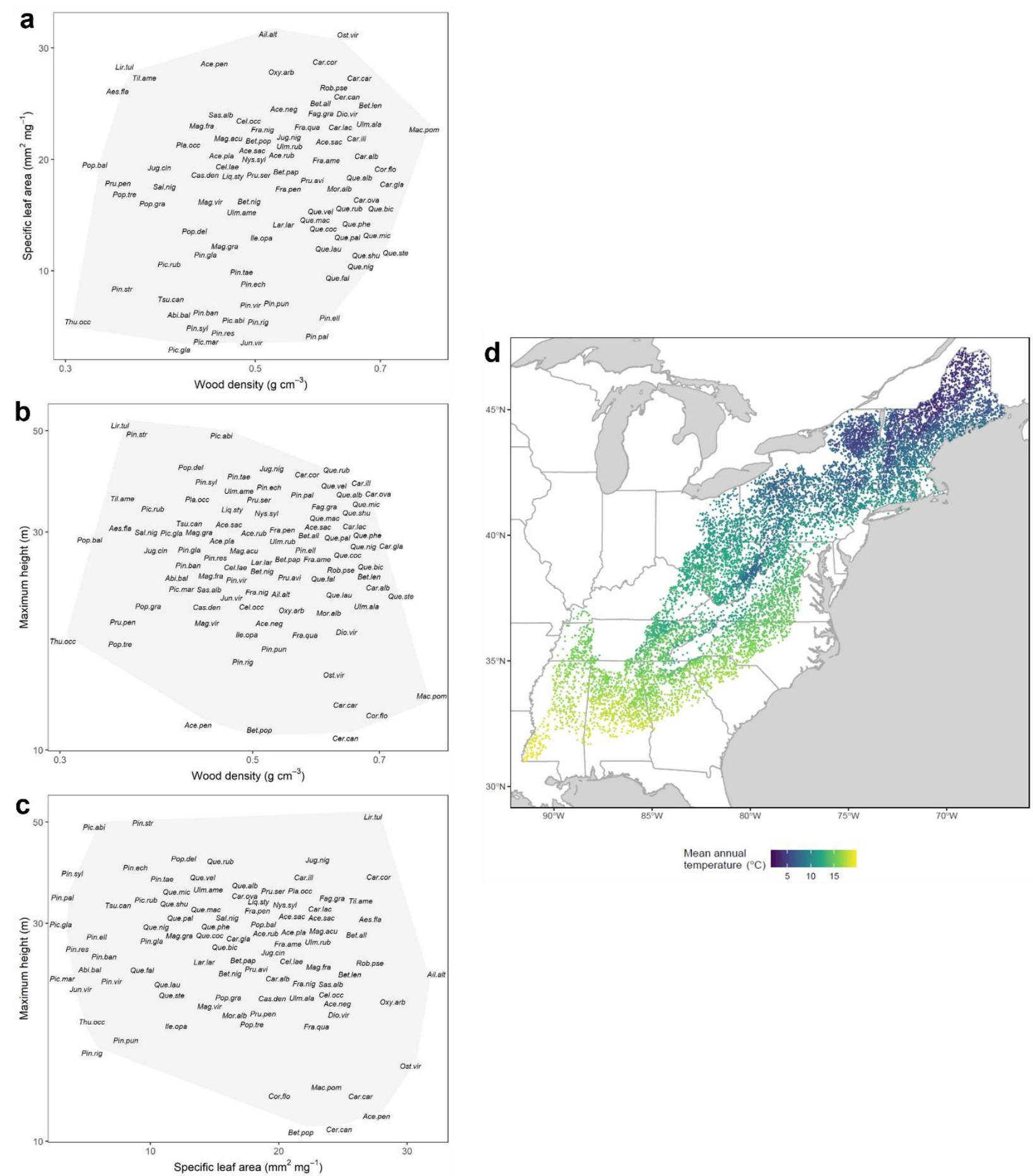

Extended Data Fig. 1 | Maps of tree species in trait space and plots in geographic space.

Trait space maps show locations of species in two-dimensional trait space defined by a) wood density and specific leaf area (SLA), b) wood density and maximum height, or c) SLA and maximum height. d) Map of US Forest Inventory and Analysis (FIA) plots included in the data set for modeling tree demographic rates. 
a

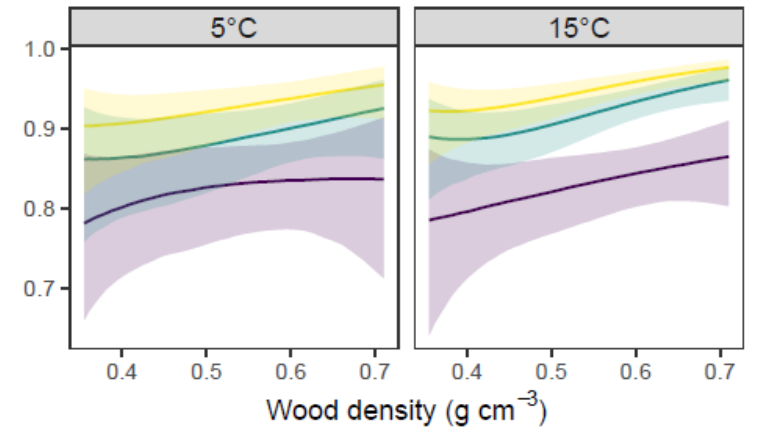

b

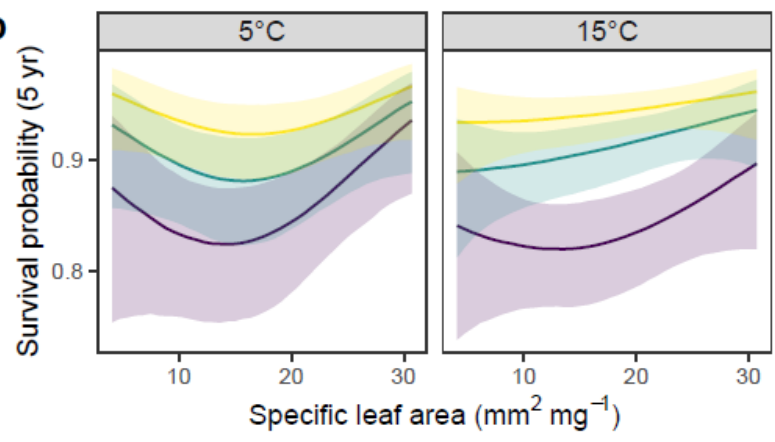

C

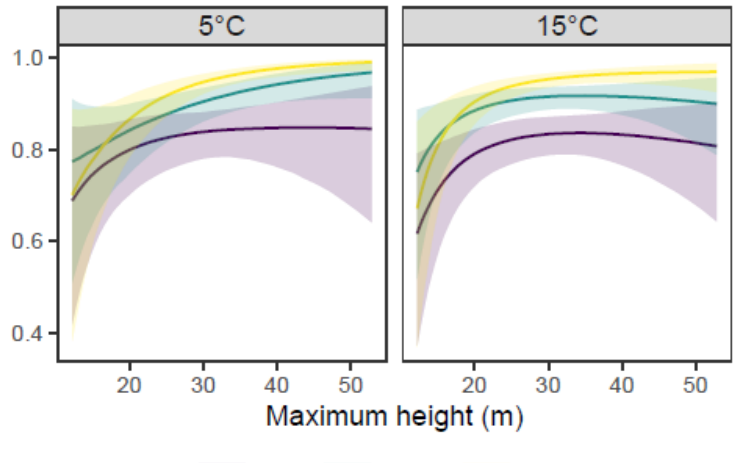

d
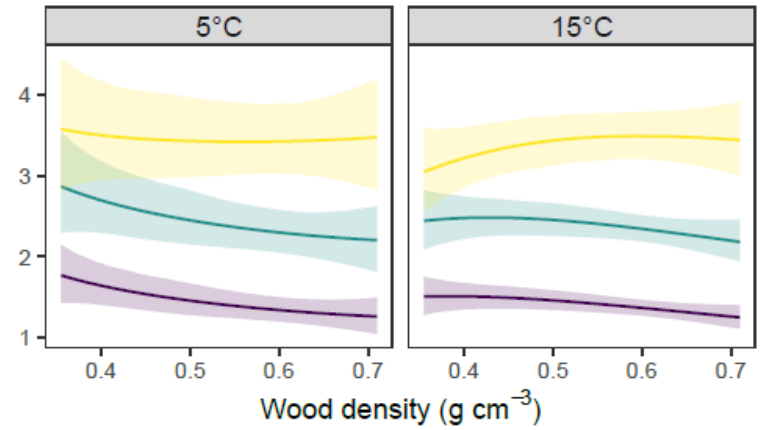

e
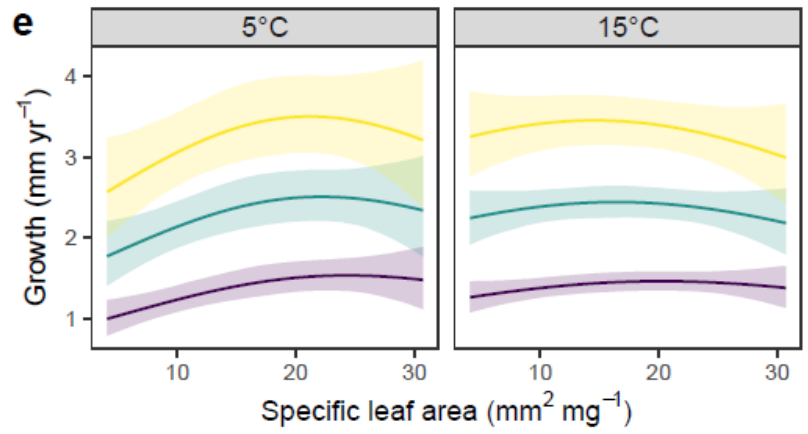

f
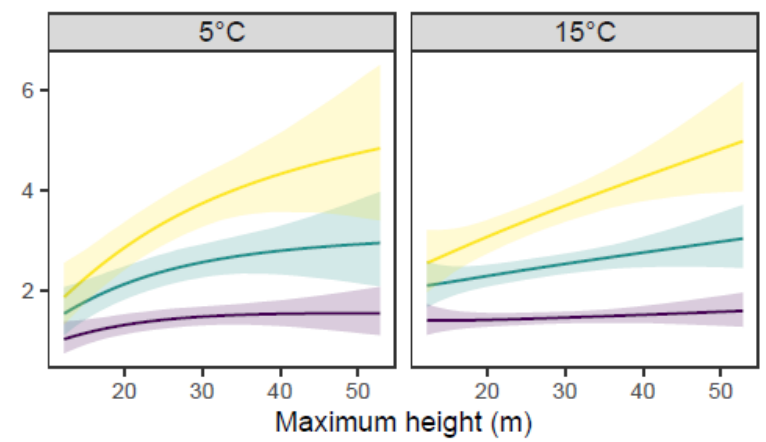

Diameter $-5 \mathrm{~cm}-15 \mathrm{~cm}-45 \mathrm{~cm}$

Extended Data Fig. 2 | Functional trait effects on survival and growth with respect to tree diameter and mean annual temperature. Plots show conditional effects of wood density $(\mathrm{a}, \mathrm{d})$, specific leaf area $(b, e)$, or maximum height $(\mathrm{c}, \mathrm{f})$ on 5-year survival probability $(\mathrm{a}-\mathrm{c})$ or diameter growth rate (d-f) at low $\left(5^{\circ} \mathrm{C}\right)$ and high $\left(15^{\circ} \mathrm{C}\right)$ mean annual temperature with other traits and crowding effects held constant at their average values. Trend lines show posterior expectations and shaded areas show $90 \%$ credible intervals. 
a

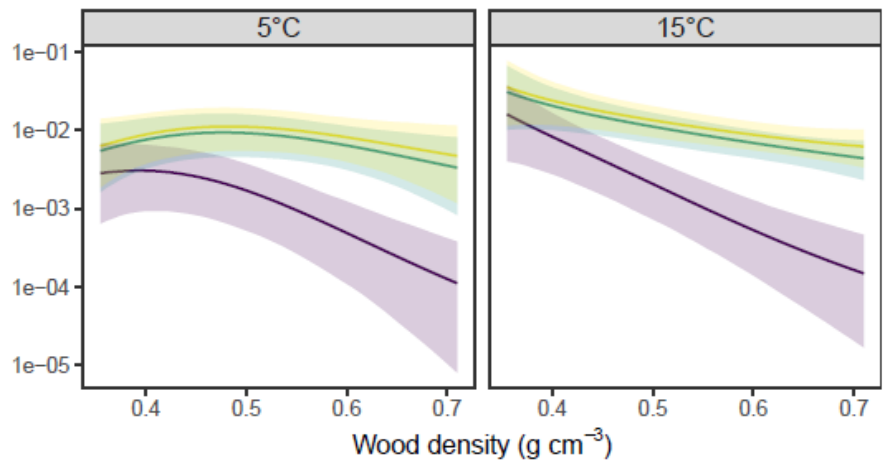

b

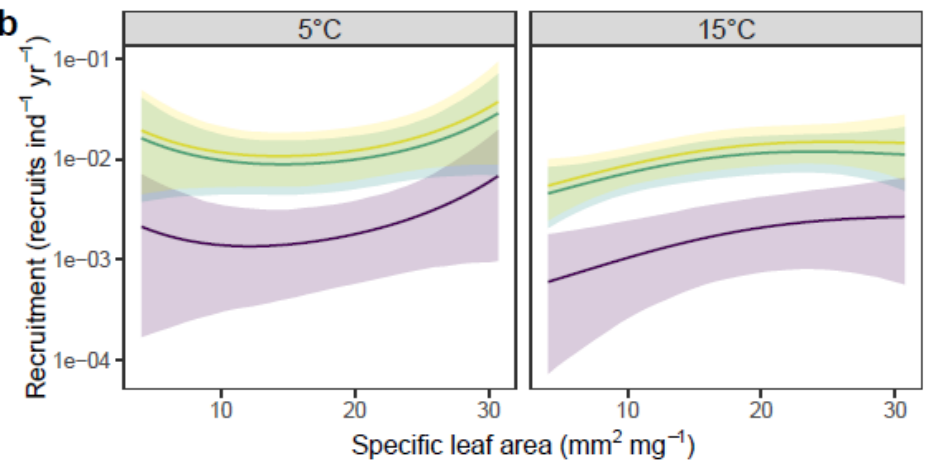

C

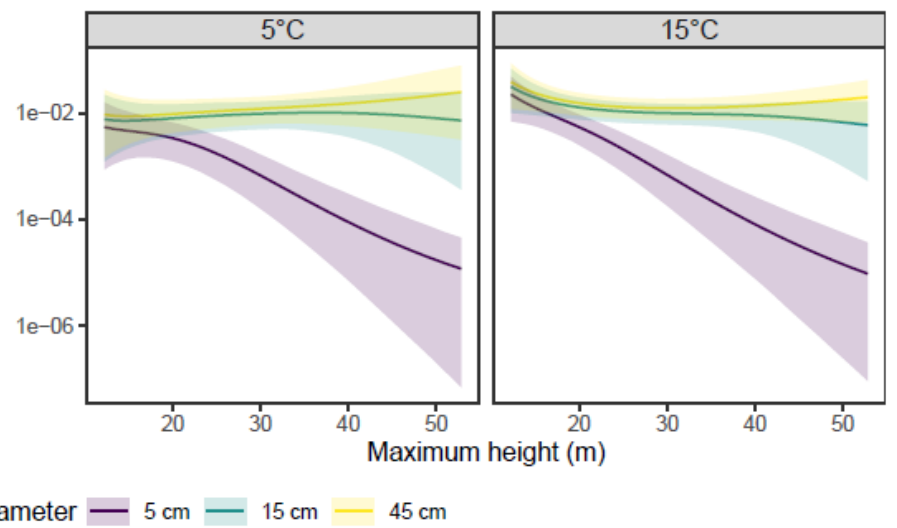

Extended Data Fig. 3 | Trait effects on recruitment with respect to tree diameter and mean annual temperature. Plots show conditional effects of wood density (a), specific leaf area (b), or maximum height (c) on recruitment rate (number of recruits, i.e., saplings reaching $2.54 \mathrm{~cm}$ diameter, produced by an adult tree per year) at low $\left(5^{\circ} \mathrm{C}\right)$ and high $\left(15^{\circ} \mathrm{C}\right)$ mean annual temperature with the other traits and crowding effects held constant at their average values. Trend lines show posterior expectations and shaded areas show $90 \%$ credible intervals. The yaxis is $\log$ transformed to show trends more clearly. 

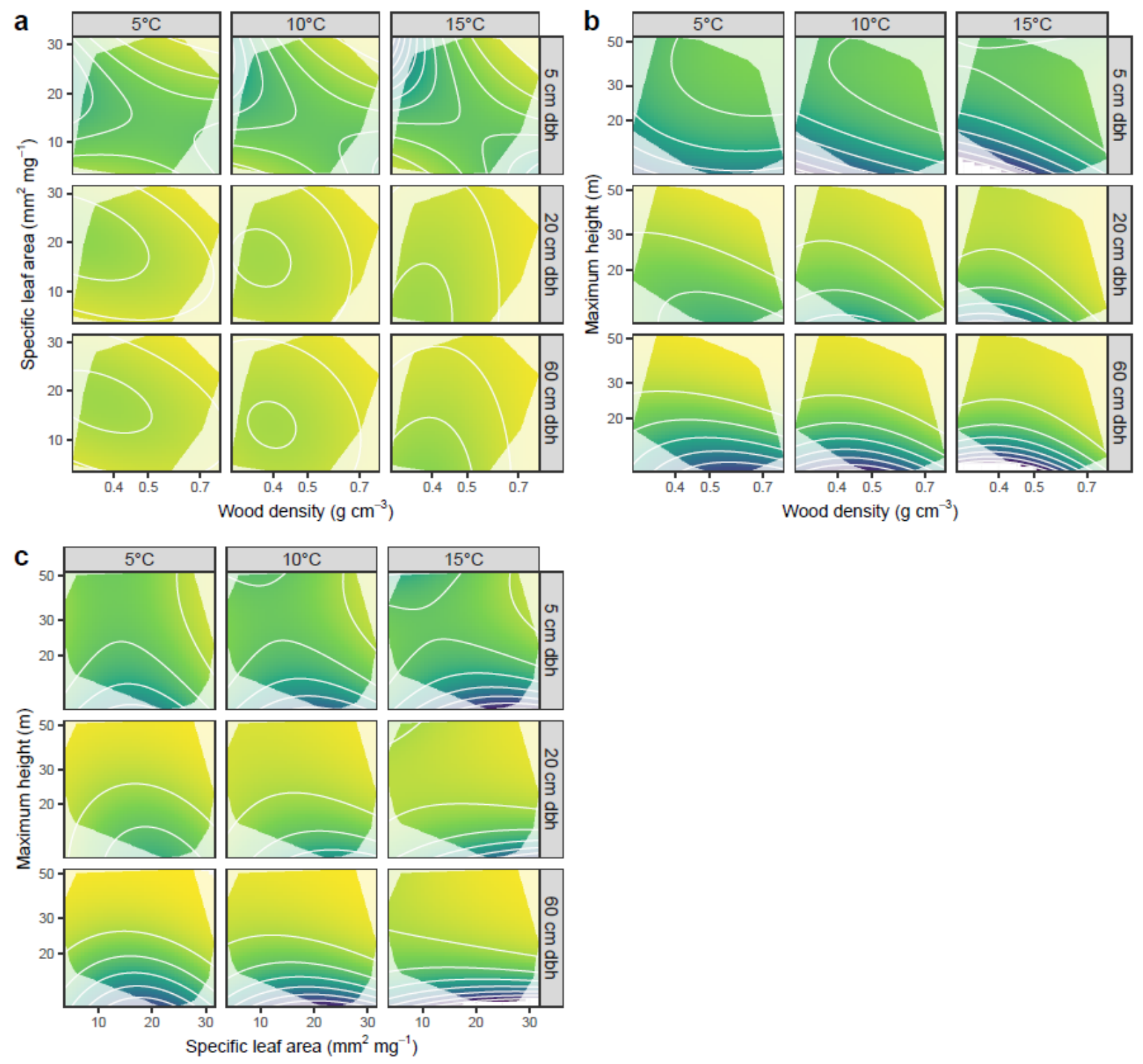

Survival probability $\left(\mathrm{yr}^{-1}\right)$

$0.90 \quad 0.95$

\section{Extended Data Fig. 4 | Survival performance landscapes with respect to tree diameter and} mean annual temperature. Landscapes show expected 1-year survival probability for trees species with different combinations of trait values. Our survival model included three traitswood density, specific leaf area (SLA), and maximum height — but for ease of visualization, landscapes are shown for two traits at a time (wood density and SLA, a; wood density and maximum height, b; SLA and maximum height, c) with the third trait held constant at its average value. Grayed areas show regions of trait space not occupied by tree species in our data set. 

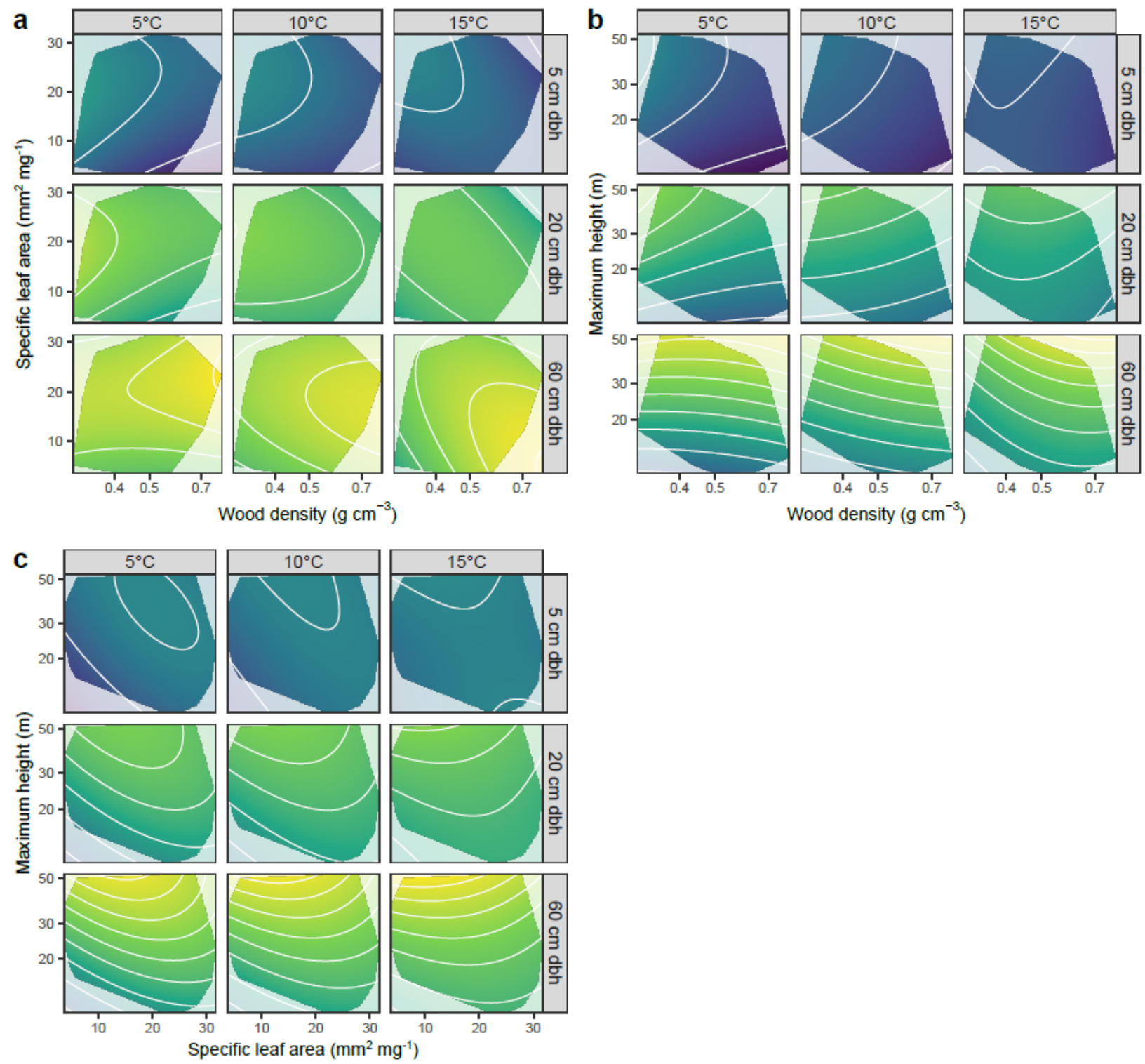

Diameter growth rate $\left(\mathrm{mm} \mathrm{yr}^{-1}\right)$

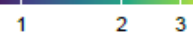

Extended Data Fig. 5 | Growth performance landscapes by tree size and temperature.

Landscapes show expected diameter growth rate for trees species with different combinations of trait values. Our growth model included three traits—-wood density, specific leaf area (SLA), and maximum height — but for ease of visualization, landscapes are shown for two traits at a time (wood density and SLA, a; wood density and maximum height, b; SLA and maximum height, c) with the third trait held constant at its average value. Grayed areas show regions of trait space not occupied by tree species in our data set. 

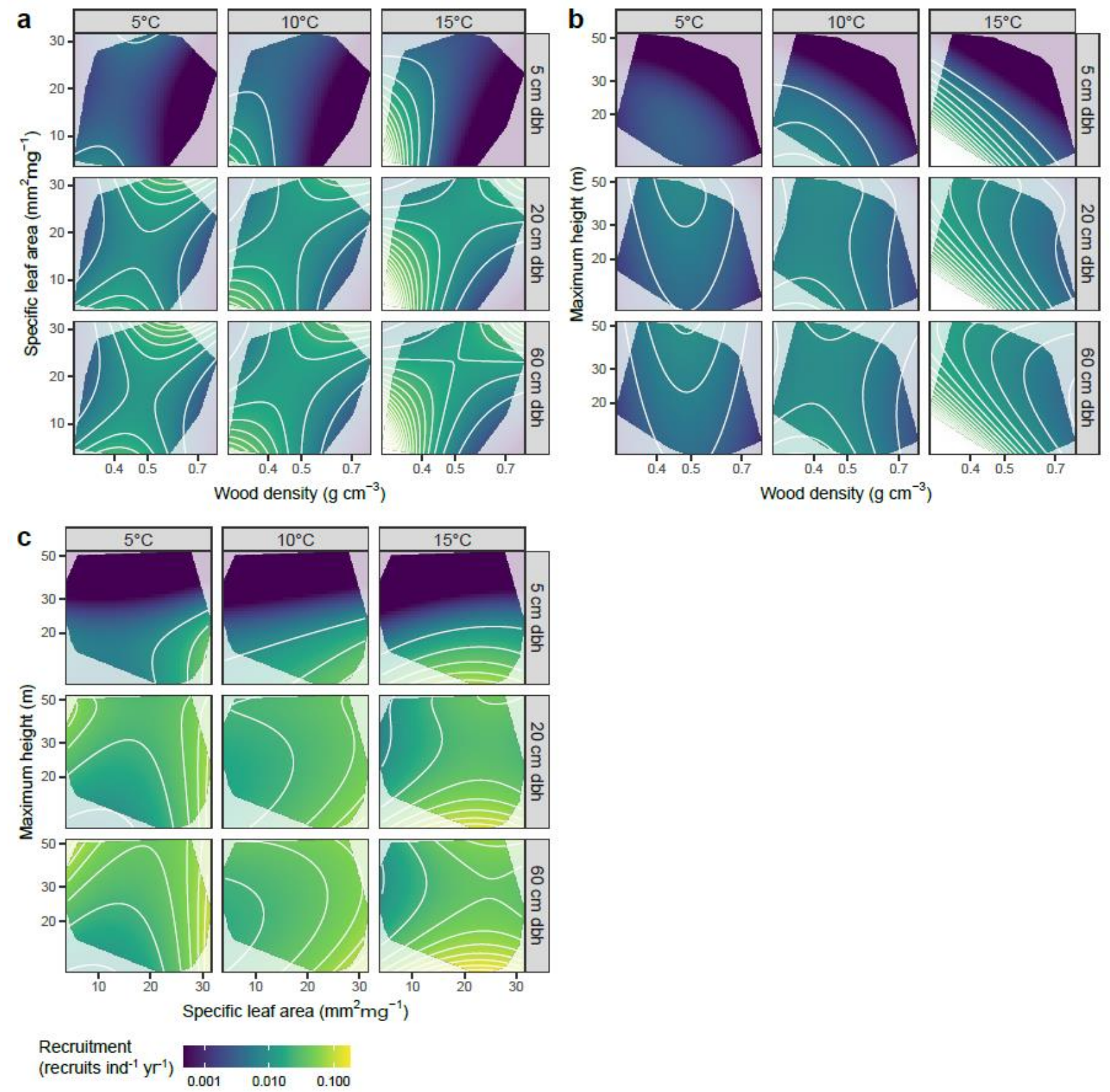

Extended Data Fig. 6 | Recruitment performance landscapes by tree diameter and mean annual temperature. Landscapes show expected recruitment rate (number of recruits, i.e., saplings reaching $2.54 \mathrm{~cm}$ diameter, produced by an adult tree per year) for trees species with different combinations of trait values. Our growth model included three traits-wood density, specific leaf area (SLA), and maximum height — but for ease of visualization, landscapes are shown for two traits at a time (wood density and SLA, a; wood density and maximum height, b; SLA and maximum height, c) with the third trait held constant at its average value. Grayed areas show regions of trait space not occupied by tree species in our data set. 

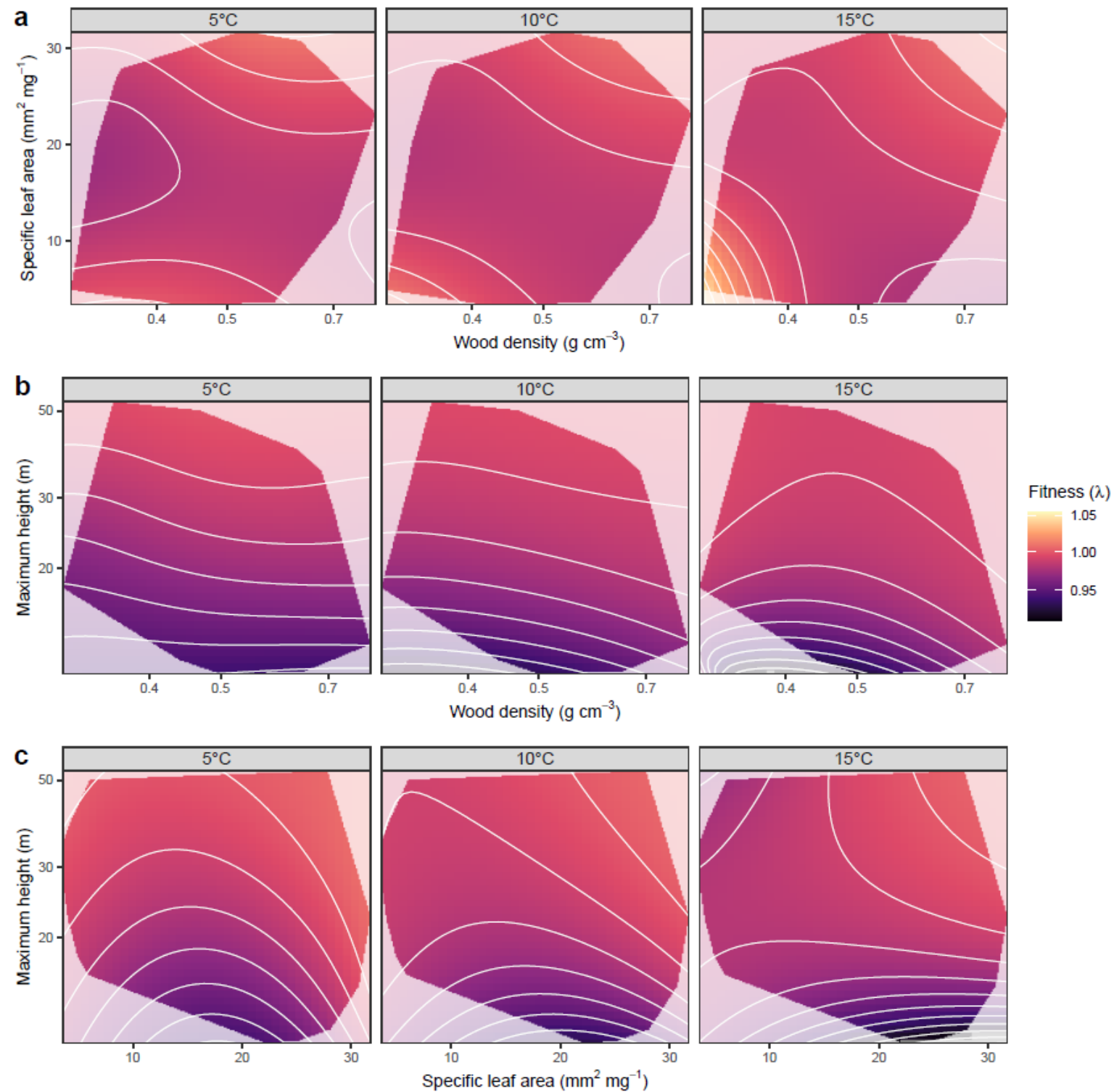

\section{Extended Data Fig. $7 \mid$ Fitness landscapes at low, medium, and high mean annual}

temperature. Landscapes show expected fitness (population growth rate, $\lambda$ ) for trees species with different trait combinations at low $\left(5^{\circ} \mathrm{C}\right)$, medium $\left(10^{\circ} \mathrm{C}\right)$, and high $\left(15^{\circ} \mathrm{C}\right)$ mean annual temperatures and average neighbor density. Models included three traits- wood density, specific leaf area (SLA), and maximum height — but for ease of visualization, landscapes are shown for two traits at a time (wood density and SLA, a; wood density and maximum height, b; SLA and maximum height, $c$ ) with the third trait held constant at its average value. Grayed areas show regions of trait space not occupied by tree species in our data set. 
a
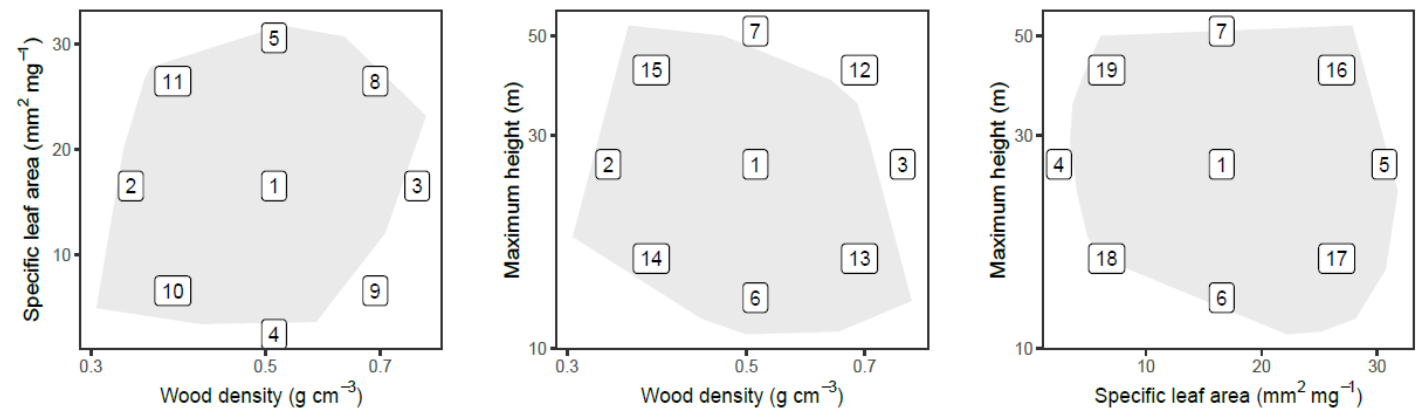

b

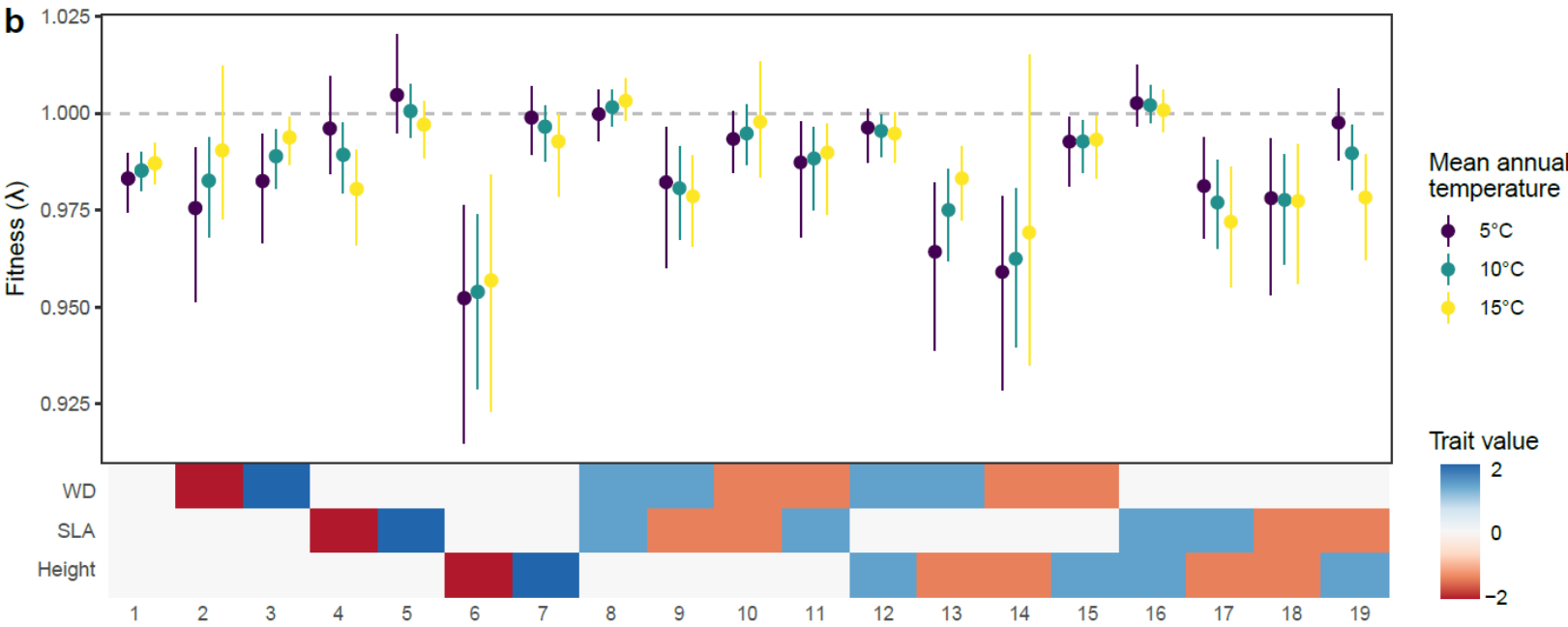

Extended Data Figure 8 | Uncertainty in fitness estimates for trait combinations in low, medium, and high mean annual temperature. To illustrate uncertainty in estimates of fitness (population growth rate, $\lambda$ ) from trait-based population projection models, we obtained fitness estimates for representative trait combinations (indicated by numbered points in trait space, panel a) at low $\left(5^{\circ} \mathrm{C}\right)$, medium $\left(10^{\circ} \mathrm{C}\right)$, and high $\left(15^{\circ} \mathrm{C}\right)$ mean annual temperatures and average neighbor density. For each trait and temperature combination, we took 1000 samples from the posterior distributions of demographic rate (survival, growth, and recruitment) models. Then, for each set of sampled demographic parameters, we constructed a population projection model (IPM) and calculated $\lambda$, resulting in a posterior distribution for $\lambda$. Points and error bars in panel $b$ show posterior means and $90 \%$ credible intervals, respectively, from this distribution. Numbered trait combinations shown on the $X$-axis of panel b correspond to numbered locations in trait space in panel a. Trait values, indicated by colored boxes below the $X$-axis of panel $\mathrm{b}$, are scaled to mean of 0 and standard deviation of 1 to make relative values comparable across traits. 

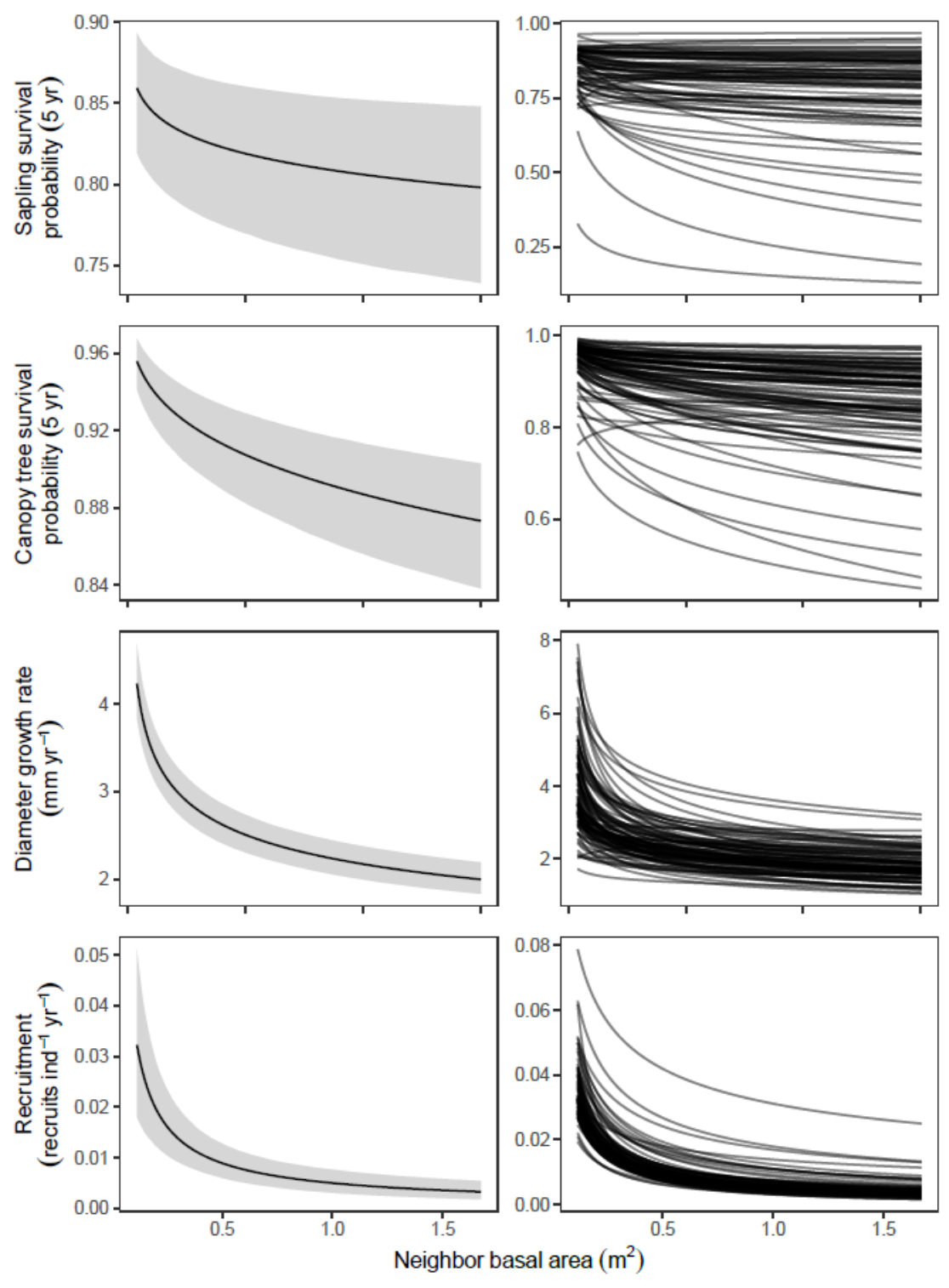

Extended Data Fig. 9. Effects of crowding on tree demographic rates. Plots show conditional mean effects (posterior mean expected values and 90\% credible intervals) and species-specific effects (right panel) of crowding (measured as total basal area of neighboring canopy trees within a plot) on tree demographic rates, with other predictors (traits, tree diameter, temperature) held constant at their average values. 

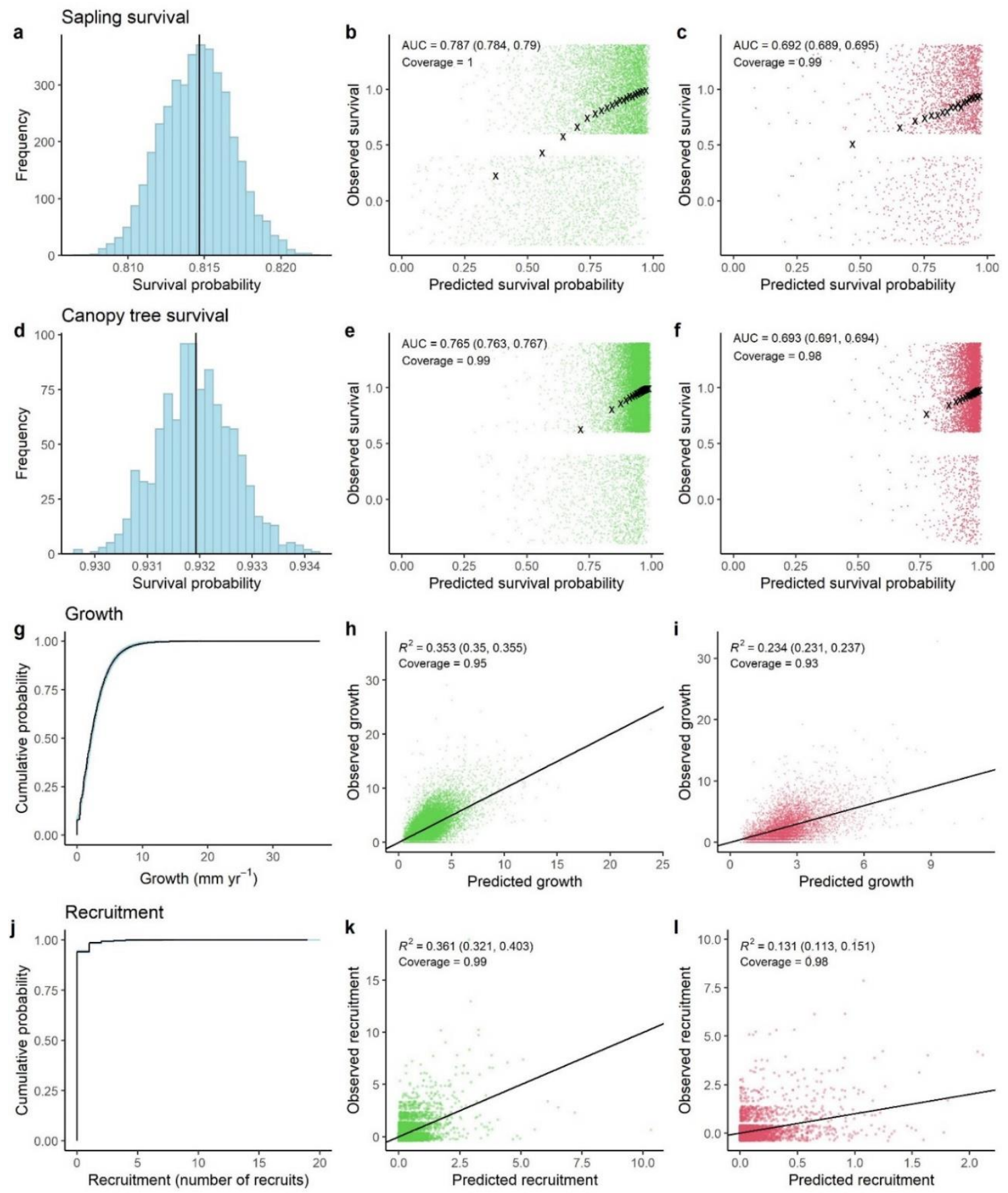

Extended Data Figure 10 | Posterior checks and model validation for demographic models. Posterior predictive checks for survival show the posterior distribution of mean survival across all trees in the data set. Posterior predictive checks for growth $(\mathrm{g})$ and recruitment $(\mathrm{j})$ show empirical cumulative distributions for observed data and posterior predictions. Panels b,e,h,j and c,f,i,l show the relationship between predicted (posterior means) and observed values for within-sample and out-of-sample data (20\% of plots), respectively. Model fit for survival was assessed using area under the ROC curve (AUC; values displayed are posterior mean and $90 \% \mathrm{CI}$ ) and coverage (proportion of observations that fall within the $90 \% \mathrm{CI}$ for predictions). Observed survival was jittered for clarity. Black x's show mean predicted and observed survival for 20 equal-sized bins. Model fit for growth and recruitment were assessed using Bayesian $\mathrm{R}^{2}$ (values displayed are posterior mean and 90\% CI) and coverage. 